\title{
Phytochemical Constituents of Combretum Loefl. (Combretaceae)
}

\author{
Amadou Dawe ${ }^{1, *}$, Saotoing Pierre ${ }^{2}$, David Emery Tsala ${ }^{2}$ and Solomon Habtemariam ${ }^{3}$
}

\author{
${ }^{I}$ Department of Chemistry, Higher Teachers' Training College, University of Maroua, P.O.Box 55 Maroua, Cameroon, \\ ${ }^{2}$ Department of Earth and Life Sciences, Higher Teachers' Training College, University of Maroua, P.O.Box 55 Ma- \\ roua, Cameroon, ${ }^{3}$ Pharmacognosy Research Laboratories, Medway School of Science, University of Greenwich, Cen- \\ tral Avenue, Chatham-Maritime, Kent ME4 4TB, UK
}

\begin{abstract}
Combretum is the largest and most widespread genus of Combretaceae. The genus comprises approximately 250 species distributed throughout the tropical regions mainly in Africa and Asia. With increasing chemical and pharmacological investigations, Combretum has shown its potential as a source of various secondary metabolites. Combretum extracts or isolates have shown in vitro bioactivitities such as antibacterial, antifungal, antihyperglycemic, cytotoxicity against various human tumor cell lines, anti-inflammatory, anti-snake, antimalarial and antioxidant effects. In vivo studies through various animal models have also shown promising results. However, chemical constituents and bioactivities of most species of this highly diversified genus have not been investigated. The molecular mechanism of bioactivities of Combretum isolates remains elusive. This review focuses on the chemistry of 261 compounds isolated and identified from 31 species of Combretum. The phytochemicals of interest are non-essential oil compounds belonging to the various structural groups such as terpenoids, flavonoids, phenanthrenes and stilbenoids.
\end{abstract}

Keywords: Combretum, phytochemistry, pharmacology, terpenoids, polyphenolic compounds, antibacterial activity, antifungal activity.

\section{INTRODUCTION}

The Combretaceae family consists of as many as 600 species of trees, shrubs and lianas in about 18-20 genera. Plants belonging to this family are found in tropical and subtropical regions, mostly in Africa and India [1]. Five genera are commonly found in southern and western Africa, of which four are tree species including the largest two genera, Combretum Loefl. and Terminalia L. [2].

Combretum comprise of about 250 species and distributed throughout the tropics and subtropics. Combretum is absent from Australia and the Pacific Islands. Three subgenera are recognized by Excell and Stace; they are Combretum, Cacoucia (Aubl.) Excell \& Stace and the monotypic Asian subgenus, Apetalantum Excell \& Stace. On the worldwide scale the subgenera Combretum and Cacoucia are separable with certainty only on the character of the presence of either scale (subgen. Combretum) or microscopic stalked glandular hairs (subgen. Cacoucia) [3]. The species of Combretum (Fig. 1) are trees, shrubs, shrublets or woody climbers, very rarely subherbaceous. Scales (subgen. Combretum) or microscopic (sometimes macroscopic) stalked glands (subgen. Cacoucia) are present. The subgenus Combretum is sometimes, in addition, divided into eleven sections, based on the floral, scale and fruit anatomy [3]. In the genus Combretum the leaves are opposite, verticillate or rarely alternate, usually petiolate, almost always with entire margins. The petiole

*Address correspondence to this author at the Department of Chemistry, Higher Teachers' Training College, University of Maroua. P.O.Box 55 Maroua, Cameroon; Tel.: + 237-94-45-73-25; Fax: +237-22-29-31-12;

E-mail: amadawe@yahoo.fr is sometimes persistant, and especially in climbers it forms a hooked wooded spine when the leaf abscises. The flowers are hermaphroditic, regular or slightly zygomorphic, 4-5merous and they are borne in elongated or subcapitate axillary or extra-axillary spikes or racemes or in terminal or terminal and axillary, often leafy panicles. The receptacle is usually clearly divided into a lower part (lower receptacle) surrounding and adnate to the ovary, and an upper receptacle which sometimes is differentiated into a lower part containing the disk and an often more expanded upper part. Sepals are 4-5 (rarely more), deltate to subulate or filiform, sometimes scarcely developed. Petals are 4-5, small and inconspicuous or showy (white, purple, red) and exceeding the sepals. Stamens are twice as many as the petals, inserted in 1 or 2 series inside the upper receptacle. The disk of the receptacle is glabrous or hairy, with or without a free margin, sometimes inconspicuous and absent. The fruit is 4-5 winged and ridged or angled, sessile or stipitate, indehiscent or rarely dehiscent; the pericarp is usually thin and papery, sometimes leathery, more rarely fleshy. Even if the fruits are often used as a good species identification characters, species identification is not always easy at the fruiting stage [3].

At least twenty four species of Combretum are well known in African traditional medicine, and used for the treatment of a variety of ailments and diseases, ranging from scorpion and snake bites, mental problems, heart and worm remedies to fever and microbial infections [4]. All parts of the Combretum species, in some cases even the fruits are used for medicinal purposes [5]. The fruits and seeds are, although, in general considered poisonous by traditional healers in various African countries and have been reported to give toxic effects on humans [6]. Combretum species are 
prepared for herbal remedies as hot water decoctions, cold water extracts or mixed with food, such as maize porridge. Sometimes fresh leaf sap is used as such. The remedies made from Combretum species are used both internally and externally. Sometimes the curing compounds of the plants are inhaled through fumes of steam baths of hot water extracts or from the smoke of burnt plant material. Dressings and ointments of different plant parts are used mainly for the treatment of wounds and infections on the skin. It is very common to mix different species of Combretum or to mix Combretum spp. with other medicinal plants for herbal remedies [3].

Several plants of the genus Combretum have been reported for their biological activities. Antibacterial activity of different extracts (ethanol, chloroform, methanol or water) of C. micranthum was noted against Pseudomonas aeruginosa, Staphylococcus aureus, Salmonella species, Streptococcus species, Proteus vulgaris, Klebsiella species, Sarcina lutea, Micrococcus luteus and Bacillus subtilis [7]. Antifungal activity against Candida albicans, antiviral activity against Herpes simplex 1 and Herpes simplex 2, antimalarial activity against Plasmodium falciparum and antidiabetic activity was also reported [8-11]. C. molle has also demonstrated antibacterial, antifungal, anthelmintic, antiasthmatic and antitussive activities [12-14]. Extracts of $C$. erythrophyllum obtained with different solvents (acetone, hexane, chloroform, carbon tetrachloride and butanol) have shown antibacterial activity at different doses against Escherichia coli, P. aeruginosa, $S$. aureus and Enterococcus faecalis [15, 16]. Moreover, in studies evaluating antifungal activity, extracts obtained with different solvents (acetone, hexane, dichloromethane and methanol) was active against the following species: $C$. albicans, C. neoformans, A. fumigatus, S. schenckii and M. canis [8].

As referenced above, there are several studies describing the bioactivities of extracts and isolated compounds from the species of the genus Combretum. Although many species of Combretum have not been extensively investigated for their chemical constituents, various classes of secondary metabolites including terpenoids, flavonoids, stilbenoids and phenanthenes have been reported. Existing phytochemical investigations indicated the presence of triterpenoids from $C$. molle, C. nigricans Lepr., C. quadrangulare, C. petrophilum Retief, $C$. edwardsii Exell, $C$. elaeagnoides Klotzsch., $C$. nelsonii Dümmer, C. bracteatum (Laws.) Engl. et Diels, $C$. laxum Jacq., C. micranthum, C. imberbe, C. padoides Engl. $\&$ Diels, C. leprosum Mart., C. sundaicum Miquel, C. oliviforme Chao, C. Zeyheri Sond., C. vendee A.E.van Wyk., $C$. erythrophyllum, C. coccineum (Sonn.) Lam. and C. rotundifolium Rich. [17-49]. Other classes of compounds isolated from Combretum include, flavonoids from C. quadrangulare, C. micranthum, C. erythrophyllum, C. apiculatum Sond., C. yannanense Exell, $C$. lanceolatum Pohl., and $C$. leprosum [23-38, 44, 51-54], diarylpropanes, phenanthrenes and stilbenoids from $C$. yannanense, $C$. griffithii Van Heurck \& Müll. Arg., C. molle, C. apiculatum Sond., C. psidioides Welw., C. Hereroense Schinz, C. cafrum (Eckl. \& Zeyh.) Kuntze and C. woodii Duemmer [39, 45-54]; galic acid derivatives from $C$. quadrangulare, C. yannanense and C. Krausii; ellagitannins from C. molle and C. glutinosum
Perr. ex DC.; and, cyclobutanes from C. albopunctatum Suesseng [28, 32, 49, 65-69].

This review focuses on the chemistry and pharmacological activities of 261 non-essential oil compounds isolated from 31 species of the genus Combretum. All of the isolated compounds with their source and references are listed in (Table 1) while their chemistry under each chemical class, such as terpenoids, flavonoids and stilbenoids, is presented separately.

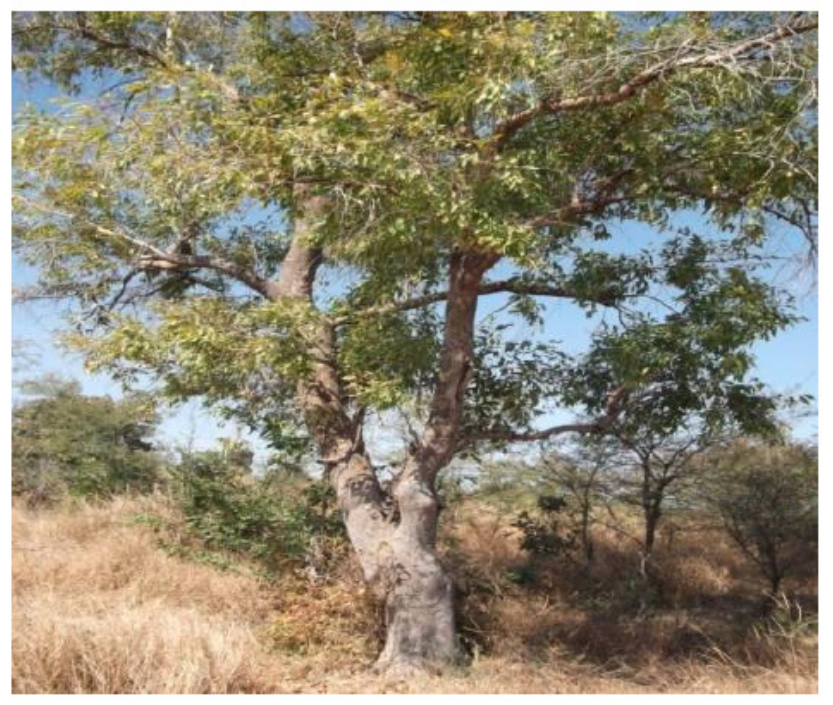

Fig. (1). Combretum fragrans (photo by Dawe Amadou).

\section{PHYTOCHEMISTRY}

Of 250 species of the genus Combretum, only 31 species (Table 1) have been more or less investigated for their phytochemistry. To date, at least 261 compounds, primarily terpenoids (mainly triterpenes) and phenolic compounds (flavonoids, stilbenoids, phenanthrenes) have been isolated and identified from these species.

\section{Triterpenoids}

Simple triterpenoids and triterpenoids glycosides are known to be the major constituents of the genus Combretum. To date, 135 compounds belonging to the cycloratane, ursanes, oleananes and dammarane triterpene and their glycosides structural groups have been isolated from the genus. Combretene A and B (1-2) were isolated from the aerial part of $C$. molle together with $2 \alpha, 3 \beta, 6 \beta$-trihydroxy-23galloylolean-12-en-28-oate (3) [17, 18]. Combregenin (4), arjungenin (5), arjunglucoside I (6), combreglucoside (7) and sericoside (8); mollic acid (9) and its glycosides [mollic acid $3 \beta$-O-glucoside (10), mollic acid 3 $\beta$-O-arabinoside (11), mollic acid $3 \beta-O$-xyloside (12)] were also reported from the stem bark of $C$. molle $[18,21]$.

Other chemical investigations led to the isolation of 73 oleananes, lupanes, cycloartanes and triterpenes glycosides types from seeds, leaves, stem bark and roots of eastern Asia $\mathrm{C}$ quadrangulare named, $1 \alpha, 3 \beta$-dihydroxy-cycloart-24ene-30-carboxylic acid (13), $1 \alpha, 3 \beta$-dihydroxy-cycloart-

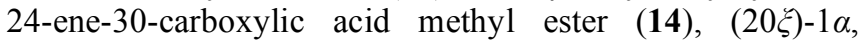
$3 \beta, 25$-trihydroxy-cycloart-21-al-23-ene-30-carboxylic acid 
Table 1. Major Non-Essential Oil Compounds Isolated and Identified from Combretum

\begin{tabular}{|c|c|c|c|}
\hline No & Compound Name & Botanical Source & Reference \\
\hline & Triterpenoids & & \\
\hline 1 & combretene A & C. molle & {$[17]$} \\
\hline 2 & combretene B & C. molle & {$[17]$} \\
\hline 3 & $2 \alpha, 3 \beta, 6 \beta$-trihydroxy-23-galloylolean-12-en-28-oate & C. molle & {$[18,54]$} \\
\hline 4 & combregenin & $\begin{array}{c}\text { C. molle } \\
\text { C. nigricans } \\
\text { C. yannanense }\end{array}$ & {$[18,19]$} \\
\hline 5 & arjungenin & $\begin{array}{c}\text { C. molle } \\
\text { C. nigricans } \\
\text { C. quadrangulare } \\
\text { C. yannanense }\end{array}$ & {$[18,19,31,54]$} \\
\hline 6 & arjunglucoside I & $\begin{array}{c}\text { C. molle } \\
\text { C. nigricans } \\
\text { C. quadrangulare } \\
\text { C. yannanense }\end{array}$ & {$[18,19,31,54]$} \\
\hline 7 & combreglucoside & $\begin{array}{c}\text { C. molle } \\
\text { C. nigricans } \\
\text { C. yannanense }\end{array}$ & {$[18,19,31]$} \\
\hline 8 & sericoside & C. molle & {$[20]$} \\
\hline 9 & mollic acid & $\begin{array}{c}\text { C. molle } \\
\text { C. nigricans } \\
\text { C. petrophilum }\end{array}$ & {$[21,22,36,39]$} \\
\hline 10 & mollic acid $3 \beta$ - $O$-glucoside & $\begin{array}{c}\text { C. molle } \\
\text { C. petrophilum }\end{array}$ & {$[21,22]$} \\
\hline 11 & mollic acid $3 \beta$-O-arabinoside & $\begin{array}{c}\text { C. molle } \\
\text { C. petrophilu } \\
\text { C. edwardsii }\end{array}$ & {$[21,22,23]$} \\
\hline 12 & mollic acid $3 \beta$ - $O$-xyloside & $\begin{array}{c}\text { C. molle } \\
\text { C. petrophilum }\end{array}$ & {$[21,22]$} \\
\hline 13 & $1 \alpha, 3 \beta$-dihydroxy-cycloart-24-ene-30-carboxylic acid & C. quadrangulare & {$[24]$} \\
\hline 14 & $1 \alpha, 3 \beta$-dihydroxy-cycloart-24-ene-30-carboxylic acid methyl ester & C. quadrangulare & {$[24]$} \\
\hline 15 & 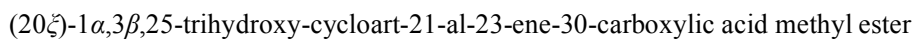 & C. quadrangulare & {$[24]$} \\
\hline 16 & quadrangularic acid $\mathrm{F}$ & C. quadrangulare & {$[25]$} \\
\hline 17 & quadrangularic acid $\mathrm{J}$ & C. quadrangulare & {$[25]$} \\
\hline 18 & quadrangularic acid $\mathrm{G}$ & C. quadrangulare & {$[25]$} \\
\hline 19 & methyl quadrangularate I & C. quadrangulare & {$[25]$} \\
\hline 20 & 24-epiquadrangularic acid M & C. quadrangulare & {$[25]$} \\
\hline 21 & 24-epiquadrangularic acid G & C. quadrangulare & {$[25]$} \\
\hline 22 & quadrangularic acid $\mathrm{M}$ & C. quadrangulare & {$[25]$} \\
\hline 23 & quadrangularic acid $\mathrm{H}$ & C. quadrangulare & {$[25]$} \\
\hline 24 & quadrangularic cid $\mathrm{K}$ & C. quadrangulare & {$[25]$} \\
\hline 25 & quadrangularic acid L & C. quadrangulare & {$[25]$} \\
\hline 26 & 24-epiquadrangularic acid L & C. quadrangulare & {$[25]$} \\
\hline
\end{tabular}


(Table 1) contd....

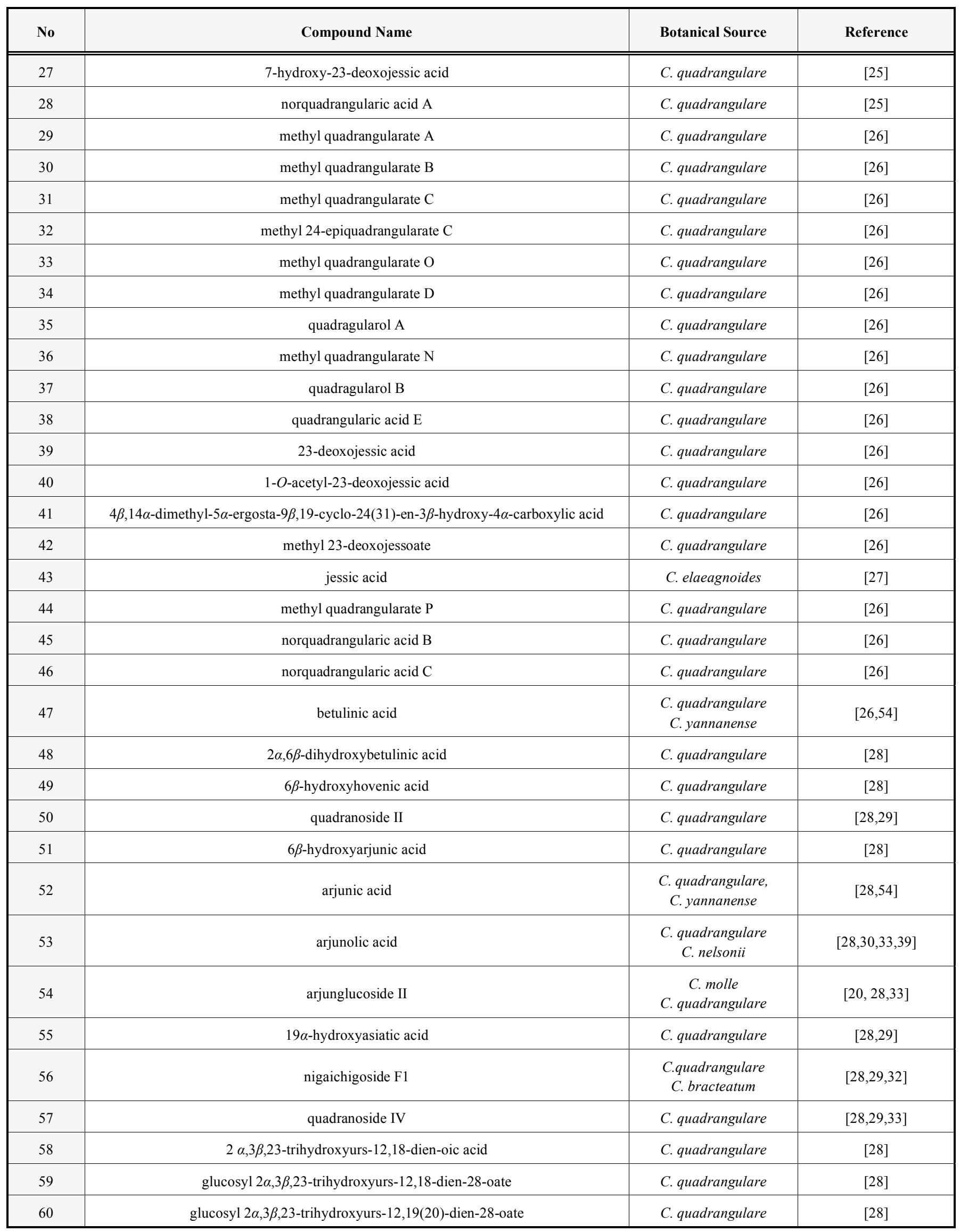


(Table 1) contd....

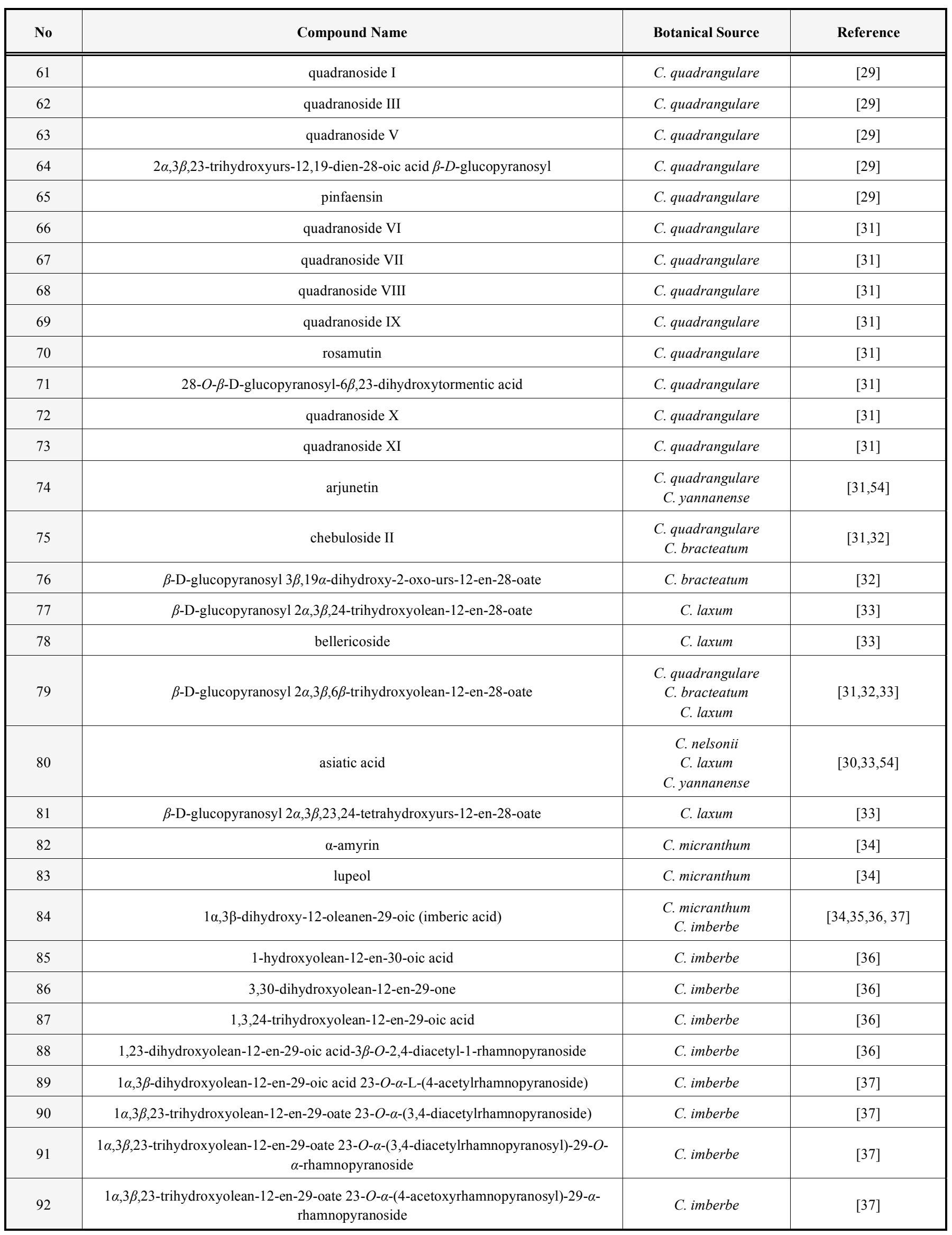


(Table 1) contd....

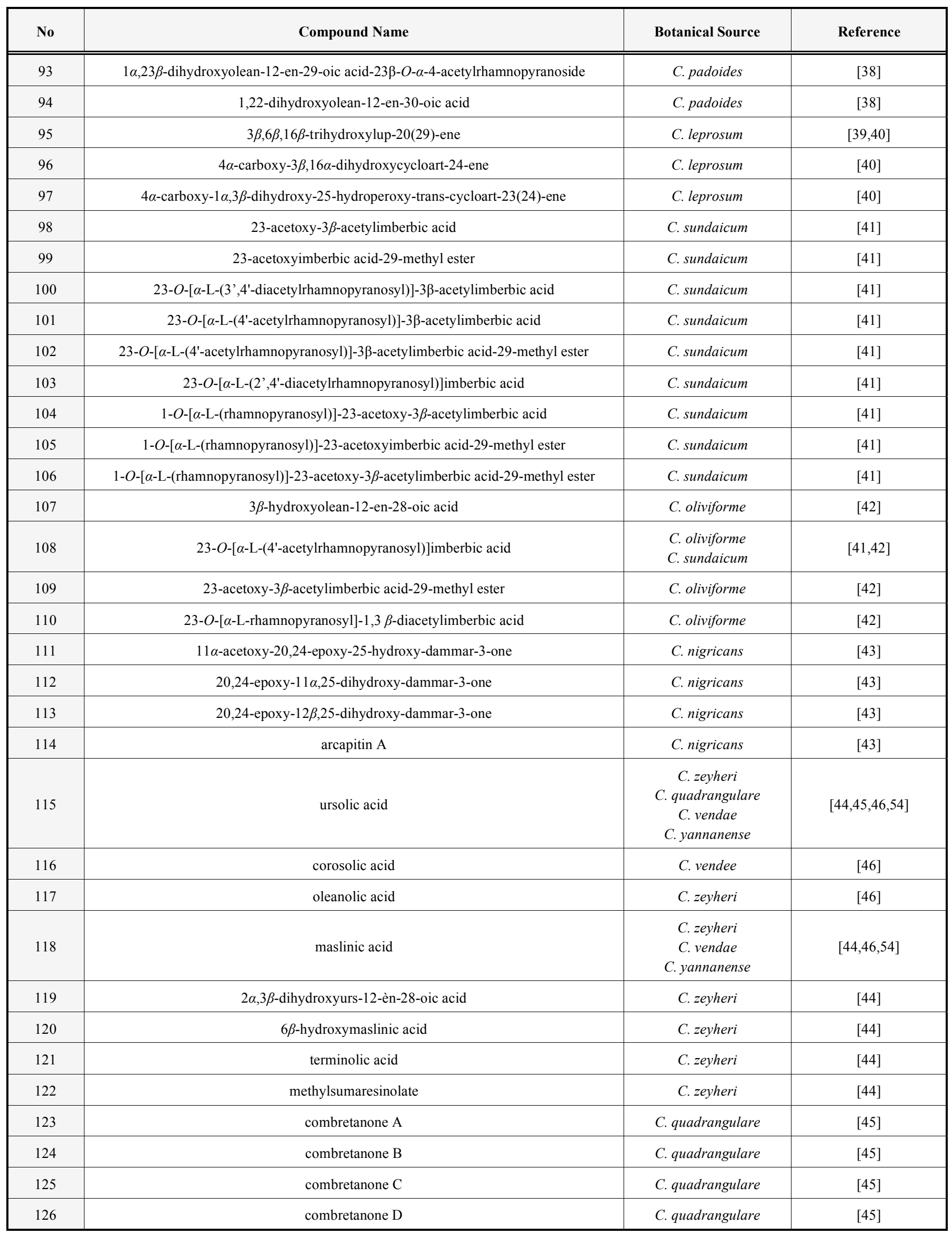


(Table 1) contd....

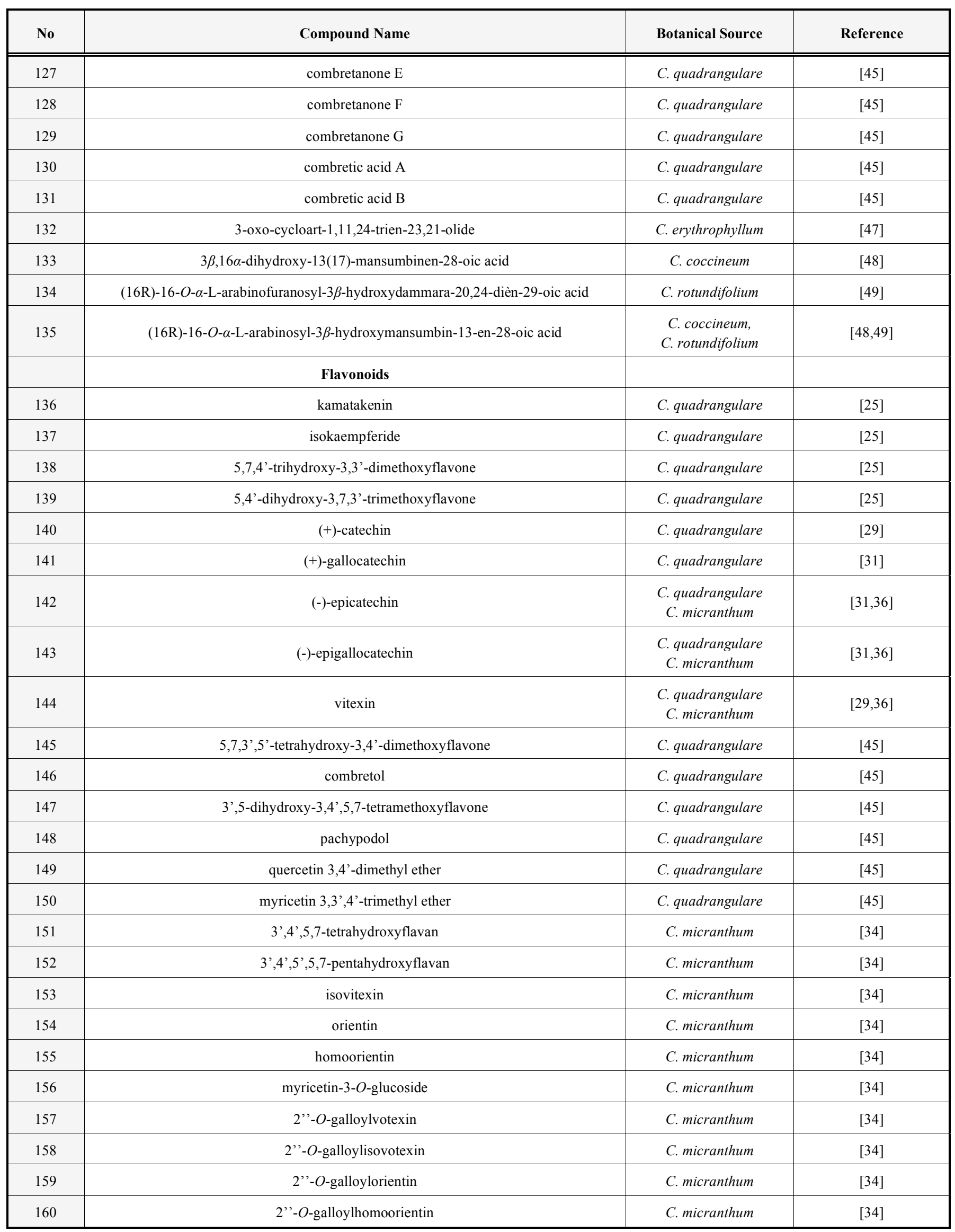


(Table 1) contd....

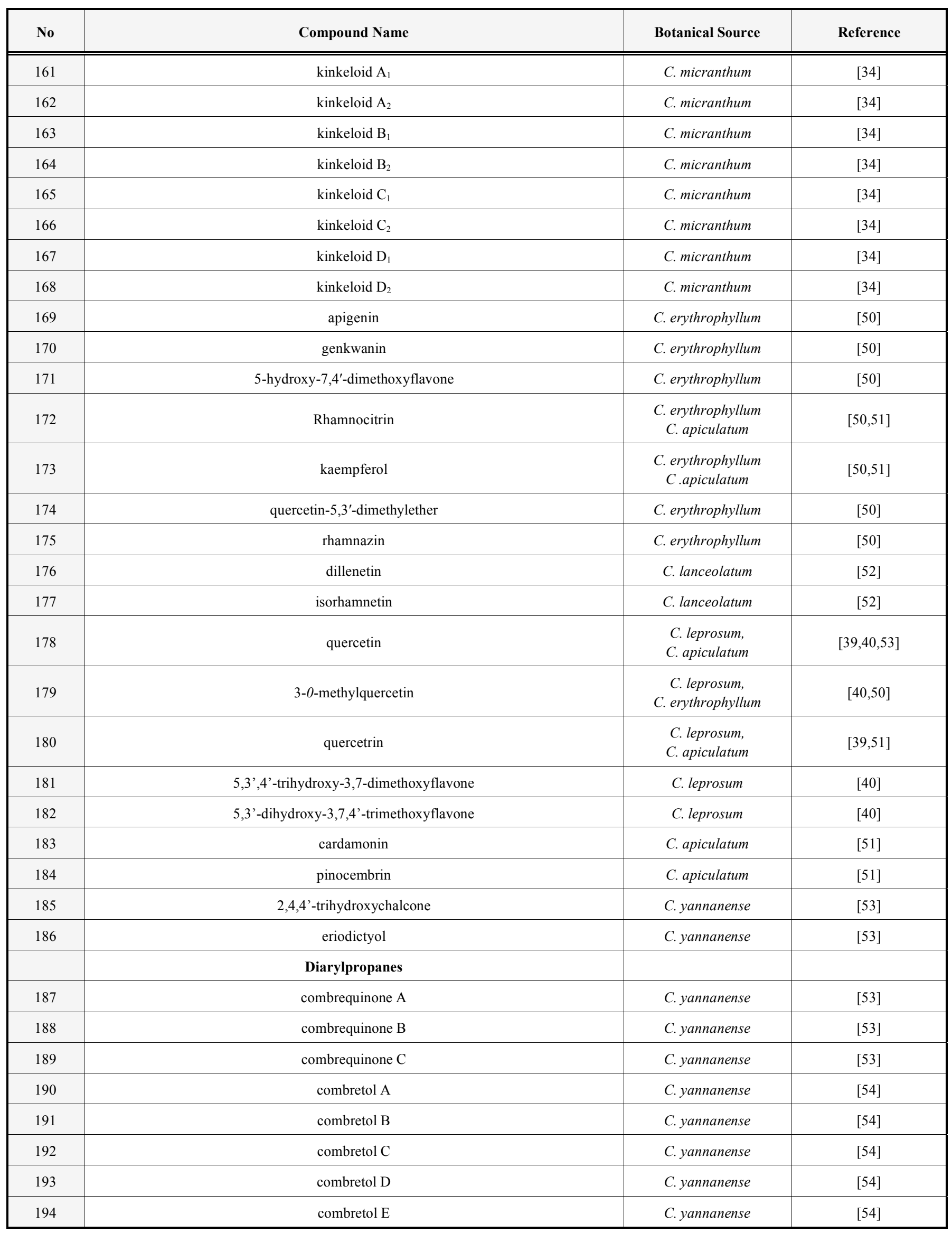


(Table 1) contd....

\begin{tabular}{|c|c|c|c|}
\hline No & Compound Name & Botanical Source & Reference \\
\hline 195 & 1-(2-methoxy-4-hydroxyphenyl) 3-(3-hydroxy-4-methoxy phenyl)-propane & C. yannanense & {$[53]$} \\
\hline 196 & 1-(4-hydroxy-2,5-dimethoxyphenyl)-3-(4-hydroxy-3-methoxyphenyl)propane & C. griffithii & {$[55]$} \\
\hline 197 & 1-(3-hydroxy-2,4-dimethoxyphenyl)-3-(4-hydroxy-3-methoxyphenyl)propane & C. griffithii & [55] \\
\hline 198 & 1-(4,5-dihydroxy-2-methoxyphenyl)-3-(3,4-dimethoxyphenyl)propane & C. griffithii & [55] \\
\hline 199 & 1-(2-hydroxy-4-methoxyphenyl)-3-(4-hydroxy-3-methoxyphenyl)propane & C. griffithii & [55] \\
\hline \multirow[t]{2}{*}{200} & 1-[2-(5-methoxy-1,4-benzoquinone)] )-3-(4-hydroxy-3-methoxyphenyl)propane & C. griffithii & [55] \\
\hline & Phenanthrenes and 9,10-Dihydrophenanthrenes & & \\
\hline 201 & 4,7-dihydroxy-2,3,6-trimethoxyphenanthrene & $\begin{array}{c}\text { C. molle, } \\
\text { C. apiculatum }\end{array}$ & {$[56]$} \\
\hline 202 & 2,6-dihydroxy-3,4,7-trimethoxyphenanthrene & C. apiculatum & [56] \\
\hline 203 & 4,6,7-trihydroxy-2,3-dimethoxyphenanthrene & $\begin{array}{c}\text { C. molle } \\
\text { C. apiculatum }\end{array}$ & [56] \\
\hline 204 & 2,3,7-dihydroxy-4,6-trimethoxyphenanthrene & C. apiculatum & [56] \\
\hline 205 & 3,6,7-dihydroxy-2,4-dimethoxyphenanthrene & C.apiculatum & {$[56]$} \\
\hline 206 & 3,7-dihydroxy-2,4,6-trimethoxyphenanthrene & C. apiculatum & {$[56]$} \\
\hline 207 & 7-hydroxy-2,4,6-trimethoxyphenanthrene & C. psidioides & [56] \\
\hline 208 & 7-hydroxy-2,3,4,6-tetramethoxyphenanthrene & $\begin{array}{l}\text { C. psidioides } \\
\text { C. cafrum }\end{array}$ & [56] \\
\hline 209 & 2,7-dihydroxy-3,4,6-trimethoxyphenanthrene & $\begin{array}{l}\text { C. psidioides } \\
\text { C. cafrum }\end{array}$ & {$[56]$} \\
\hline 210 & 2,6,7-trihydroxy-3,4-dimethoxyphenanthrene & C. psidioides & {$[56]$} \\
\hline 211 & 3,6-dihydroxy-2,4,7-trimethoxyphenanthrene & C. hereroense & [56] \\
\hline 212 & 2,6-dihydroxy-3,4,7-trimethoxy-9,10-dihydrophenanthrene & $\begin{array}{c}\text { C. molle } \\
\text { C. apiculatum }\end{array}$ & [56] \\
\hline 213 & 4,7-dihydroxy-2,6-dimethoxy-9,10-dihydrophenanthrene & C. apiculatum & {$[56]$} \\
\hline 214 & 2,6-dihydroxy-4,7-trimethoxy-9,10-dihydrophenanthrene & C. apiculatum & {$[56]$} \\
\hline 215 & 4,7-dihydroxy-2,3,6-trimethoxy-9,10-dihydrophenanthrene & $\begin{array}{c}\text { C. molle } \\
\text { C. apiculatum }\end{array}$ & {$[56]$} \\
\hline 216 & 2-hydroxy-3,4,6,7-tetramethoxy-9,10-dihydrophenanthrene & $\begin{array}{l}\text { C. apiculatum } \\
\text { C. cafrum }\end{array}$ & [56] \\
\hline 217 & 6,7-dihydroxy-2,3,4-trimethoxy-9,10-dihydrophenanthrene & $\begin{array}{l}\text { C. apiculatum } \\
\text { C. cafrum }\end{array}$ & {$[56]$} \\
\hline 218 & 4,6,7-trihydroxy-2,3-dimethoxy-9,10-dihydrophenanthrene & $\begin{array}{c}\text { C. molle } \\
\text { C. apiculatum }\end{array}$ & {$[56]$} \\
\hline 219 & 7-hydroxy-2,4,6-trimethoxy-9,10-dihydrophenanthrene & C. psidioides & {$[56]$} \\
\hline 220 & 7-hydroxy-2,3,4,6-tetramethoxy-9,10-dihydrophenanthrene & $\begin{array}{l}\text { C. psidioides } \\
\text { C. apiculatum }\end{array}$ & {$[56]$} \\
\hline 221 & 2,7-dihydroxy-3,4,6-trimethoxy-9,10-dihydrophenanthrene & $\begin{array}{l}\text { C. psidioides } \\
\text { C. apiculatum } \\
\text { C. cafrum }\end{array}$ & [56] \\
\hline 222 & 2,6,7-trihydroxy-3,4-dimethoxy-9,10-dihydrophenanthrene & C. psidioides & {$[56]$} \\
\hline 223 & 3,6-dihydroxy-2,4,7-trimethoxy-9,10-dihydrophenanthrene & C. hereroense & {$[57]$} \\
\hline
\end{tabular}


(Table 1) contd....

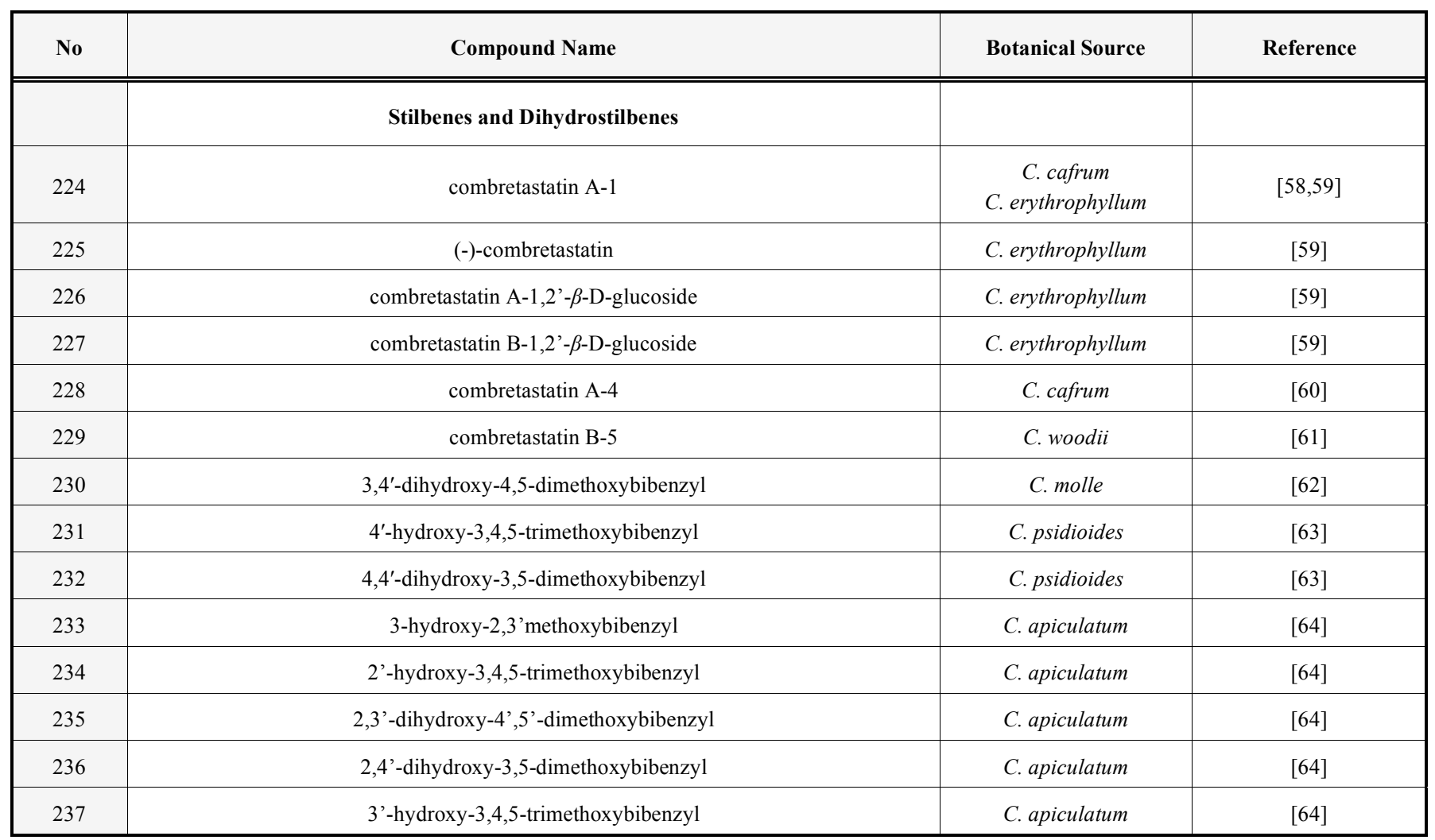

methyl ester (15), quadrangularic acid E-H (38,16,18,23), JM (17, 24, 25, 22), methyl quadrangularate A-C, D, I, N, O, $\mathrm{P}(\mathbf{2 9 - 3 1}, 34,19,36,33,44), 24$-epiguadrangularic acid $\mathrm{M}$, $\mathrm{G}, \mathrm{L}(\mathbf{2 0}, \mathbf{2 1}, \mathbf{2 6})$, norquadrangularic acid A-C (28, 44, 45), methyl 24-epiquadrangularate $C(\mathbf{3 2})$, quadrangularol $\mathrm{A}$ and B $(35,37)$, quadranoside I-XI $(61,50,62,57,63,66-69,72-$ 73), jessic acid (43), 23-deoxojesic acid (39), methyl 23deoxojessoate (42), 7-hydroxy-23-deoxojessic acid (27), 1$O$-acetyl-23-deoxojessic acid (40), arjunic acid (52), $6 \beta$ hydroxyarjunic acid (51), arjunolic acid (53), arjunglucoside II (54), arjunetin (74), arjungenin (6), asiatic acid (80), 19 $\alpha$ hydroxyasiatic acid (55), 6 $\beta$-hydroxyhovenic acid (49), betulinic acid (47), 2 $\alpha, 6 \beta$-dihydroxybetulinic acid (48), 2 $\alpha, 3 \beta, 23$-trihydroxyurs-12,18-dien-oic acid (58), glucosyl $2 \alpha, 3 \beta, 23$-trihydroxyurs-12,18-dien-28-oate (59), glucosyl $2 \alpha, 3 \beta, 23$-trihydroxyurs-12,19(20)-dien-28-oate $\quad(60)$, $2 \alpha, 3 \beta, 23$-trihydroxyurs-12,19-dien-28-oic acid $\beta$ - $D$-glucopyranosyl (64), 28-O- $\beta$-D-glucopyranosyl-6 $\beta, 23$-dihydroxytormentic acid (71), $\beta$-D-glucopyranosyl $2 \alpha, 3 \beta, 6 \beta$ trihydroxyolean-12-en-28-oate (79), $4 \beta, 14 \alpha$-dimethyl-5 $\alpha$ ergosta-9 $\beta, 19$-cyclo-24(31)-en-3 $\beta$-hydroxy- $4 \alpha$-carboxylic acid (41), ursolic acid (115), pinfaensin (65), rosamutin (70) nigaichigoside F1 (56), chebuloside II (75), combretanones A-G (123-129), combretic acid A and B (130-131) [23-30, 45].

Nine 1,3-hydroxylated pentacyclic olean-12-ene triterpenoids were isolated from the leaves of south African Combretum imberbe by preparative HPLC. Complete spectral analysis of these compounds showed that they are, imberic acid (84), 1-hydroxyolean-12-en-30-oic acid (85), 3,30dihydroxyolean-12-en-29-one (86), 1,3,24-trihydroxyolean12-en-29-oic acid (87), 1,23-dihydroxyolean-12-en-29-oic acid-3 $\beta$-O-2,4-diacetyl-1-rhamnopyranoside (88) [36]. Four other glycoside derivatives of the above triterpenoids type were also isolated from the leaves of the same species and named, $1 \alpha, 3 \beta$-dihydroxyolean-12-en-29-oic acid $23-O-\alpha$-L(4-acetylrhamnopyranoside) (89), $1 \alpha, 3 \beta, 23$-trihydroxyolean12-en-29-oate 23-O- $\alpha$-(3,4-diacetylrhamnopyranoside) (90), $1 \alpha, 3 \beta, 23$-trihydroxyolean-12-en-29-oate 23-O- $\alpha$-(3,4-diacetylrhamnopyranosyl)-29- $O$ - $\alpha$-rhamnopyranoside (91), $1 \alpha, 3 \beta$, 23-trihydroxyolean-12-en-29-oate 23-O- $\alpha$-(4-acetoxyrhamno pyranosyl)-29- $\alpha$-rhamnopyranoside (92) [37]. Another compounds with a similar squeleton $1 \alpha, 23 \beta$-dihydroxyolean-12en-29-oic acid-23 $\beta-O-\alpha$-4-acetylrhamno-pyranoside (93) was isolated from the dichloromethane extract of the leaves of South African $C$. padoides leaves together with 1,22dihydroxyolean-12-en-30-oic acid (94) [38] and C. leprosum led to the isolation of $3 \beta, 6 \beta, 16 \beta$-trihydroxylup-20(29)-ene (95), $4 \alpha$-carboxy-3 $\beta, 16 \alpha$-dihydroxycycloart-24-ene (96) and $4 \alpha$-carboxy- $1 \alpha, 3 \beta$-dihydroxy-25-hydroperoxy-trans-cycloart23(24)-ene (97) [39].

Bioassay-guided purification of the ethyl acetate extracts obtained from the leaves and flowers of Combretum sundaicum led to the isolation of 10 pentacyclic triterpenoids possessing olean-12-en-28-oic acid and olean-12-en-29-oic acid aglycons. These compounds are presented in (Table 1) and named, 23-acetoxy-3 $\beta$-acetylimberbic acid (98), 23acetoxyimberbic acid-29-methyl ester (99), 23- $O$-[ $\alpha$-L-(3', 4'diacetylrhamnopyranosyl)]-3 $\beta$-acetylimberbic acid (100), $23-O-[\alpha-\mathrm{L}-(4$ '-acetylrhamnopyranosyl) $]-3 \beta$-acetylimberbic acid (101), 23- $O$ - $[\alpha$-L-(4'-acetylrhamnopyranosyl $)]-3 \beta$ acetylimberbic acid-29-methyl ester (102), 23- $O$ - $\left[\alpha-\mathrm{L}-\left(2^{\prime}, 4^{\prime}-\right.\right.$ diacetylrhamnopyranosyl)] imberbic acid (103), 1- $O-[\alpha-\mathrm{L}-$ 


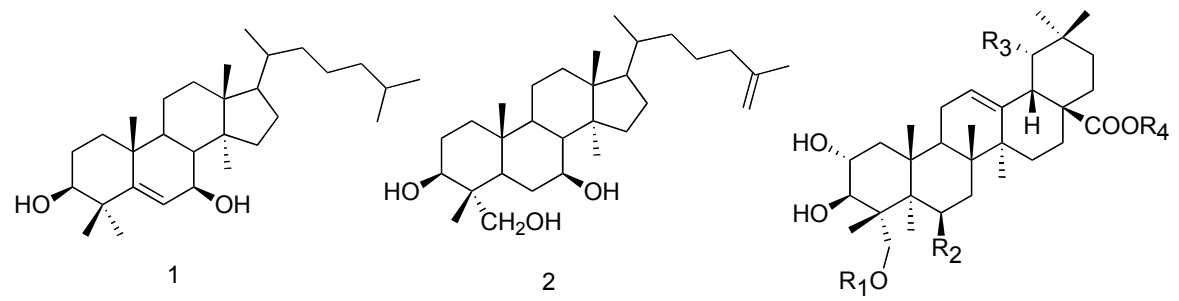

$\begin{array}{llll}3: \mathrm{R} 1=\text { galloyl } & \mathrm{R} 2=\mathrm{OH} & \mathrm{R} 3=\mathrm{H} & \mathrm{R} 4=\mathrm{glc} \\ 4: \mathrm{R} 1=\mathrm{H} & \mathrm{R} 2=\mathrm{OH} & \mathrm{R} 3=\mathrm{OH} & \mathrm{R} 4=\mathrm{H} \\ 5: \mathrm{R} 1=\mathrm{H} & \mathrm{R} 2=\mathrm{H} & \mathrm{R} 3=\mathrm{OH} & \mathrm{R} 4=\mathrm{H} \\ 6: \mathrm{R} 1=\mathrm{H} & \mathrm{R} 2=\mathrm{H} & \mathrm{R} 3=\mathrm{OH} & \mathrm{R} 4=\mathrm{glc} \\ 7: \mathrm{R} 1=\mathrm{H} & \mathrm{R} 2=\mathrm{OH} & \mathrm{R} 3=\mathrm{OH} & \mathrm{R} 4=\mathrm{glc} \\ 8: \mathrm{R} 1=\mathrm{H} & \mathrm{R} 2=\mathrm{H} & \mathrm{R} 3=\mathrm{OH} & \mathrm{R} 4=\mathrm{glc}\end{array}$

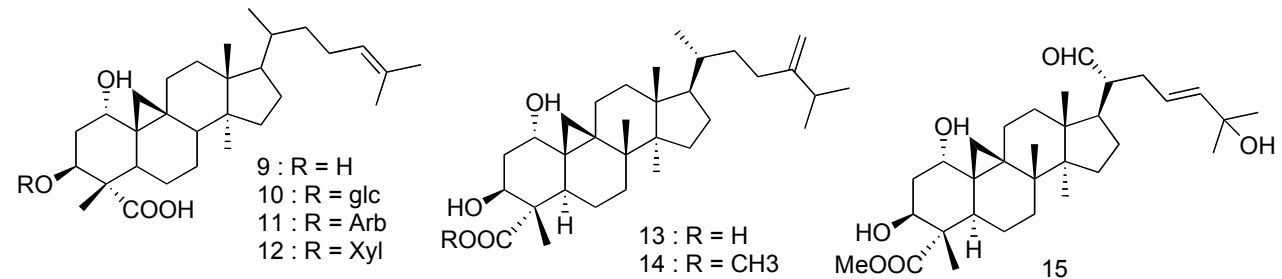

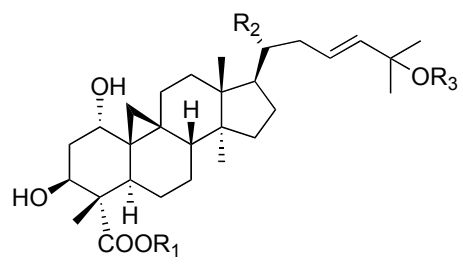

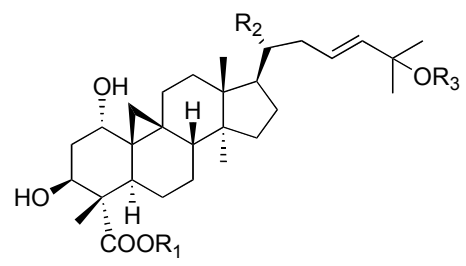

$$
\begin{array}{ll}
16: R 1=M e & R 2=\mathrm{COOH} \\
17: R 1=H & R 2=\mathrm{OH}
\end{array}
$$

16: $\mathrm{R} 1=\mathrm{Me} \mathrm{R} 2=\mathrm{COOH} \quad \mathrm{R} 3=\mathrm{OH}$ $17: R 1=H \quad R 2=R 3=M e$

$17: \mathrm{R} 1=\mathrm{H} \quad \mathrm{R} 2=\mathrm{R} 3=\mathrm{Me}$
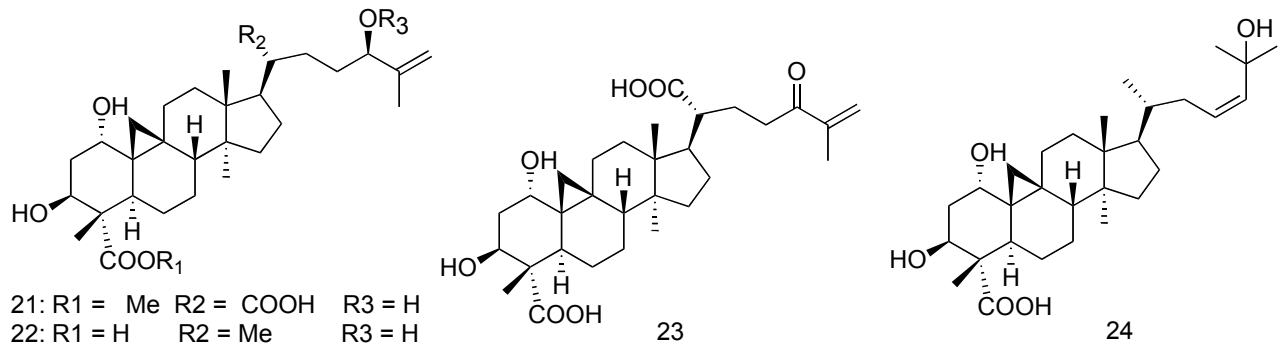

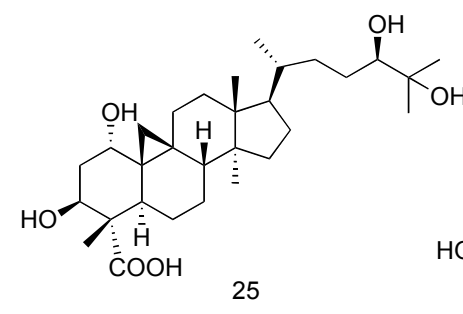

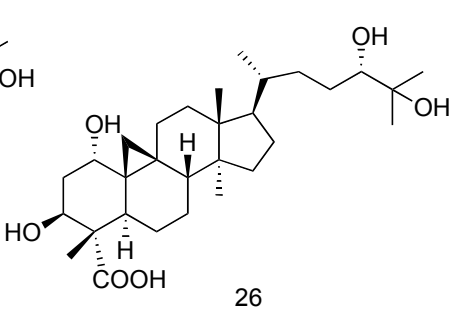

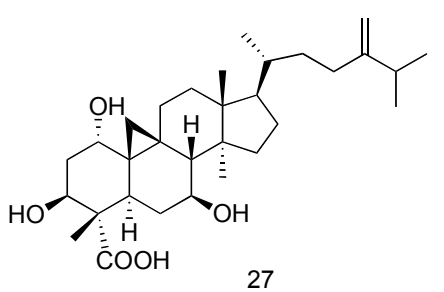

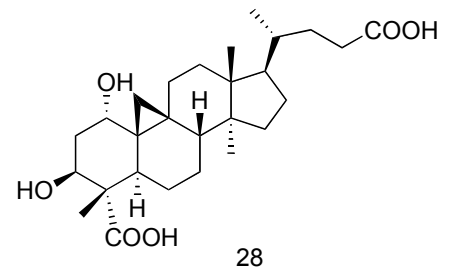

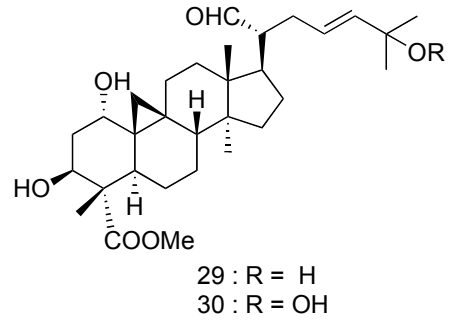




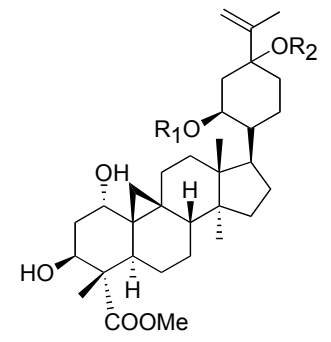

31: R1 = Me R2 = b-H

32: R1 = Me R2 = a - H

$33: \mathrm{R} 1=\mathrm{H} \quad \mathrm{R} 2=\mathrm{b}-\mathrm{H}$

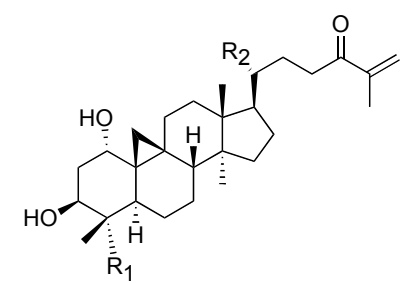

$36: \mathrm{R} 1=\mathrm{COOMe} \quad \mathrm{R} 2=\mathrm{CHO}$

$37: \mathrm{R} 1=\mathrm{CH} 2 \mathrm{OH} \quad \mathrm{R} 2=\mathrm{Me}$
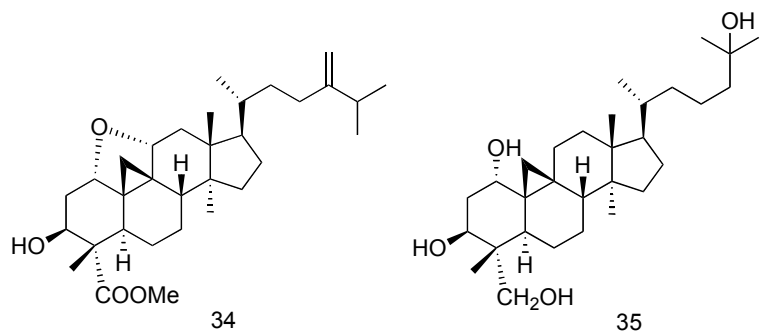

35
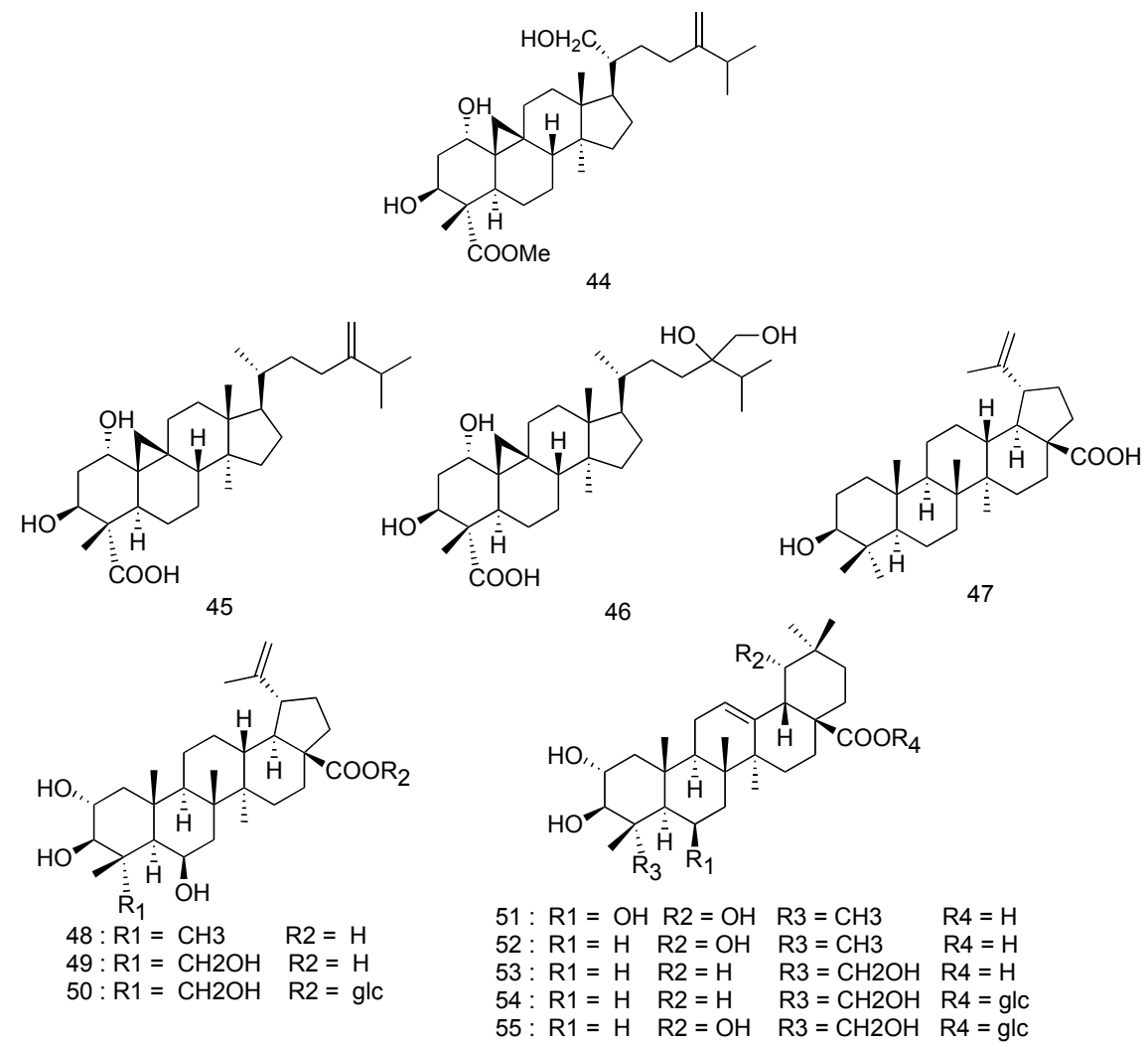

45
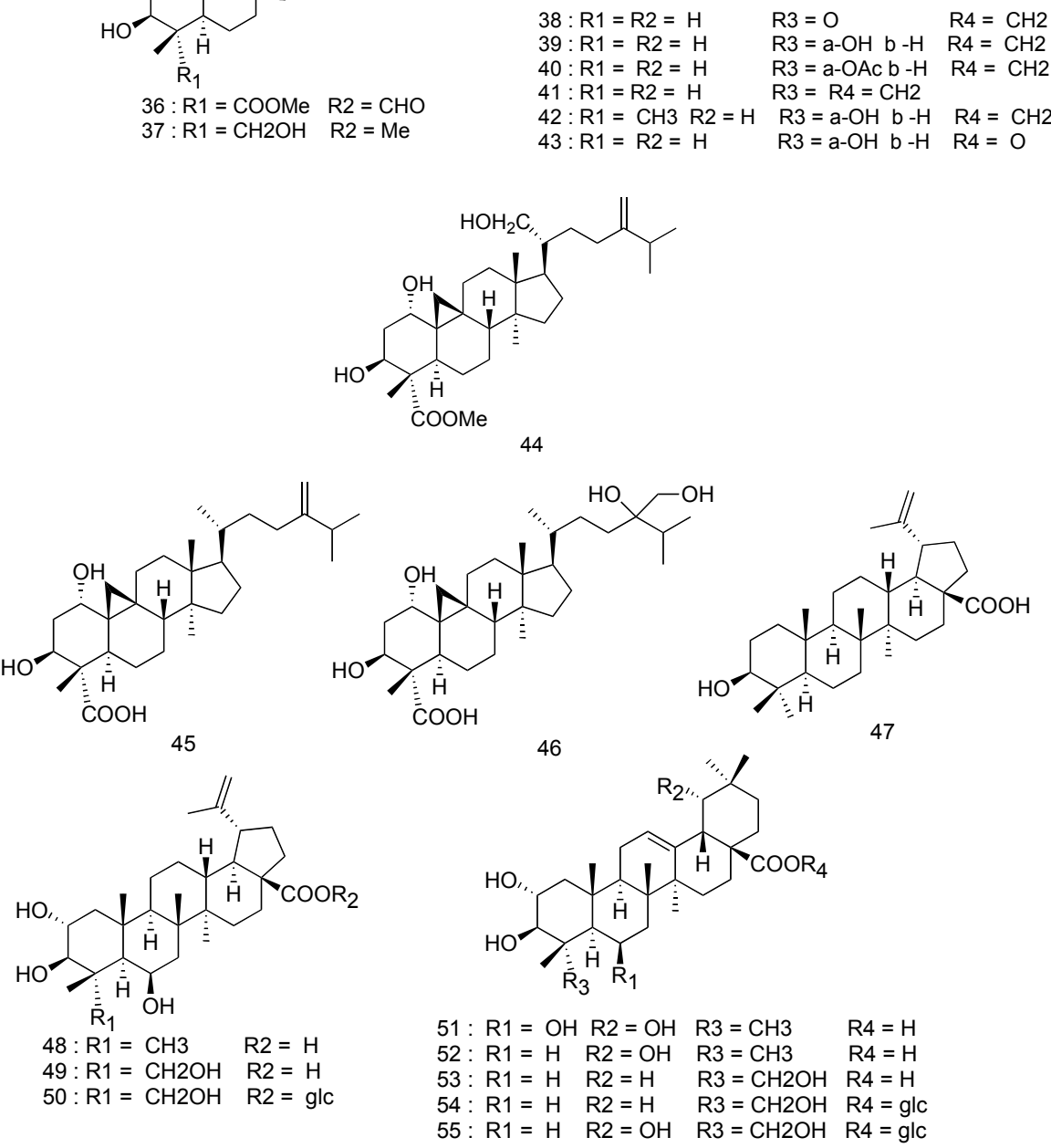

$43: \mathrm{R} 1=\mathrm{R} 2=\mathrm{H}$

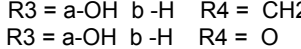

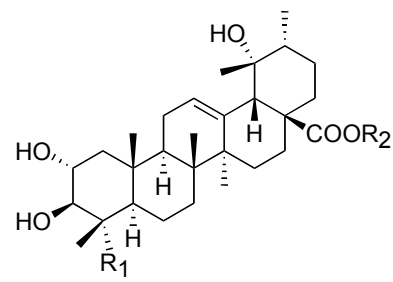

56: $\mathrm{R} 1=\mathrm{CH} 2 \mathrm{OH} \quad \mathrm{R} 2=\mathrm{H}$

57: $\mathrm{R} 1=\mathrm{CH} 2 \mathrm{OH} \quad \mathrm{R} 2=\mathrm{glc}$

58: $\mathrm{R} 1=\mathrm{CH} 3 \quad \mathrm{R} 2=\mathrm{glc}$

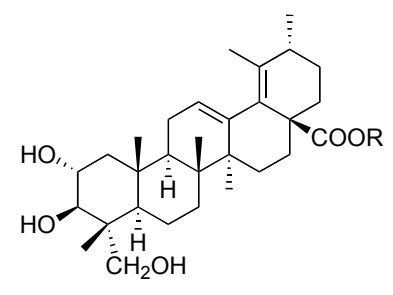

59: $\mathrm{R}=\mathrm{H}$

$60: R=g l c$

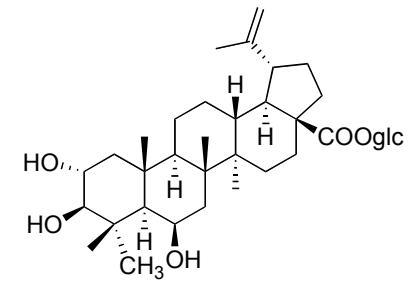

61 

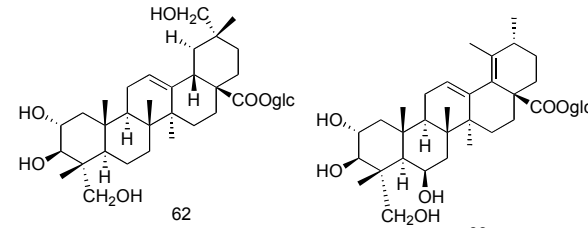

62

$\mathrm{C} \mathrm{H}_{2} \mathrm{OH}$

63

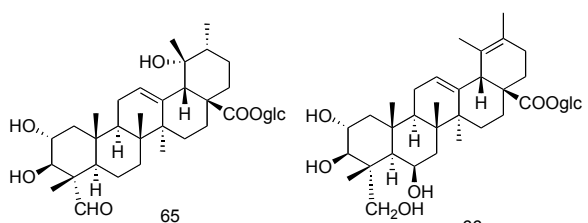

66

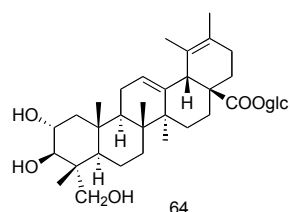

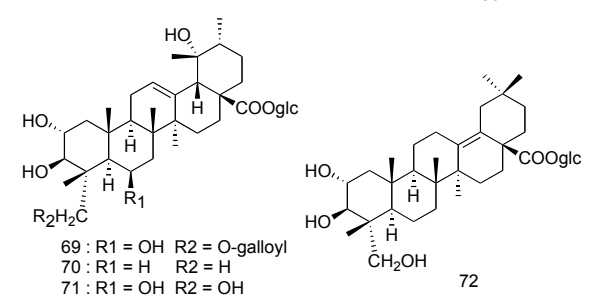

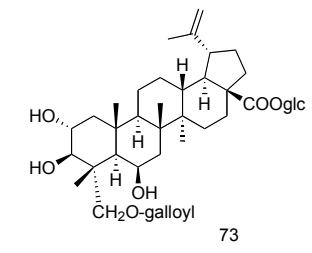

(c)

$74: \mathrm{R} 1=\mathrm{H} \quad \mathrm{R} 2=\mathrm{OH} \mathrm{R} 3=\mathrm{H}$ $75: \mathrm{R} 1=\mathrm{OH} \mathrm{R} 2=\mathrm{H} \quad \mathrm{R} 3=\mathrm{OH}$

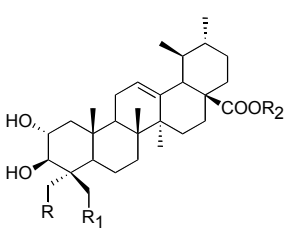

$80: \mathrm{R} 1=\mathrm{OH} \mathrm{R} 2=\mathrm{H} \quad \mathrm{R} 3=\mathrm{H}$ 81: R1 $=\mathrm{OH} \quad \mathrm{R} 2=\mathrm{OH} \quad \mathrm{R} 3=$ b-D-glc

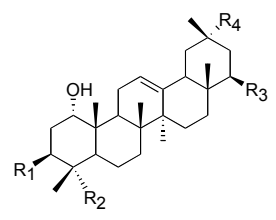

$\mathrm{R} 2=\mathrm{CH} 3 \quad \mathrm{R} 3=\mathrm{H} \quad \mathrm{R} 4=\mathrm{COOH} 89: \mathrm{R} 1=\mathrm{OAc} \mathrm{R} 2=3,4-\mathrm{Ac}-\mathrm{O}-\mathrm{Rh} \quad \mathrm{R} 3=\mathrm{COOH}$ $\begin{array}{lllll}84: \mathrm{R} 1=\mathrm{OH} & \mathrm{R} 2=\mathrm{CH} 3 & \mathrm{R} 3=\mathrm{H} & \mathrm{R} 4=\mathrm{COOH} 89: \mathrm{R} 1=\mathrm{OAc} \mathrm{R} 2=3,4-\mathrm{Ac}-\mathrm{O}-\mathrm{Rh} \mathrm{R} 3=\mathrm{COOH} \\ 85: \mathrm{R} 1=\mathrm{H} & \mathrm{R} 2=\mathrm{CH} 3 & \mathrm{R} 3=\mathrm{H} & \mathrm{R} 4=\mathrm{COOH}_{90}: \mathrm{R} 1=\mathrm{OAc} \mathrm{R} 2=4-\mathrm{AcO}-\mathrm{Rh} & \mathrm{R} 3=\mathrm{COOH} \\ 86: \mathrm{R} 1=\mathrm{H} & \mathrm{R} 2=\mathrm{CH} 3 & \mathrm{R} 3=\mathrm{O} & \mathrm{R} 4=\mathrm{CH} 2 \mathrm{OH} \\ 87: \mathrm{R} 1=\mathrm{H} & \mathrm{R} 2=\mathrm{CH} 3 & \mathrm{R} 3=\mathrm{OH} & \mathrm{R} 4=\mathrm{COOH}^{91}: \mathrm{R} 1=\mathrm{OAc} \mathrm{R} 2=4-\mathrm{AcO}-\mathrm{Rh} & \mathrm{R} 3=\mathrm{COOCH} 3 \\ 88: \mathrm{R} 1=2,4-\text { diAc-Rh } & \mathrm{R} 2=\mathrm{CH} 2 \mathrm{OH} & \mathrm{R} 3=\mathrm{H} & \mathrm{R} 4=\mathrm{COOH} 92: \mathrm{R} 1=\mathrm{OH} \quad \mathrm{R} 2=2,4-\mathrm{AcO}-\mathrm{Rh} & \mathrm{R} 3=\mathrm{COOH}\end{array}$ $\begin{array}{lllll}84: \mathrm{R} 1=\mathrm{OH} & \mathrm{R} 2=\mathrm{CH} 3 & \mathrm{R} 3=\mathrm{H} & \mathrm{R} 4=\mathrm{COOH} 89: \mathrm{R} 1=\mathrm{OAc} \mathrm{R} 2=3,4-\mathrm{Ac}-\mathrm{O}-\mathrm{Rh} R 3=\mathrm{ROOH} \\ 85: \mathrm{R} 1=\mathrm{H} & \mathrm{R} 2=\mathrm{CH} 3 & \mathrm{R} 3=\mathrm{H} & \mathrm{R} 4=\mathrm{COOH}_{90}: \mathrm{R} 1=\mathrm{OAc} \mathrm{R} 2=4-\mathrm{AcO}-\mathrm{Rh} & \mathrm{R} 3=\mathrm{COOH} \\ 86: \mathrm{R} 1=\mathrm{H} & \mathrm{R} 2=\mathrm{CH} 3 & \mathrm{R} 3=\mathrm{O} & \mathrm{R} 4=\mathrm{CH}_{2} \mathrm{OH} \\ 87: \mathrm{R} 1=\mathrm{H} & \mathrm{R} 2=\mathrm{CH} 3 & \mathrm{R} 3=\mathrm{OH} & \mathrm{R} 4=\mathrm{COOH}^{91}: \mathrm{R} 1=\mathrm{OAc} \mathrm{R} 2=4-\mathrm{AcO}-\mathrm{Rh} & \mathrm{R} 3=\mathrm{COOCH} \\ 88: \mathrm{R} 1=2,4-\text { diAc-Rh } & \mathrm{R} 2=\mathrm{CH} 2 \mathrm{OH} & \mathrm{R} 3=\mathrm{H} & \mathrm{R} 4=\mathrm{COOH}_{2}: \mathrm{R} 1=\mathrm{OH} \quad \mathrm{R} 2=2,4-\mathrm{AcO}-\mathrm{Rh} & \mathrm{R} 3=\mathrm{COOH}\end{array}$

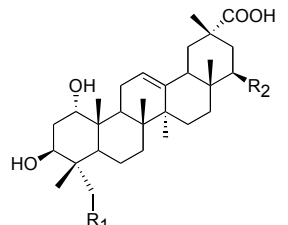

93: $\mathrm{R} 1=\mathrm{OAC}-\mathrm{Rh} \quad \mathrm{R} 2=\mathrm{H}$

$94: \mathrm{R} 1=\mathrm{H} \quad \mathrm{R} 2=\mathrm{OH}$

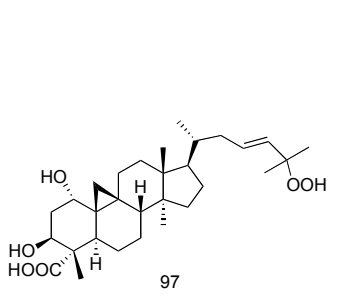

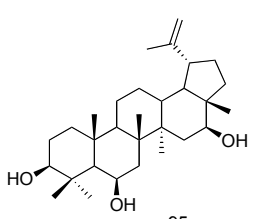

95

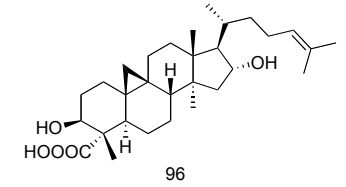

96

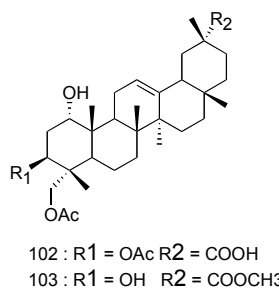


(rhamnopyranosyl)]-23-acetoxy-3 $\beta$-acetylimberbic acid (104), 1- $O$-[ $\alpha$-L-(rhamnopyranosyl)]-23-acetoxyimberbic acid-29-methyl ester (105), 1-O-[ $\alpha$-L-(rhamnopyranosyl)]23 -acetoxy-3 $\beta$-acetylimberbic acid-29-methyl ester (106), 23-O-[ $\alpha$-L-(4'-acetylrhamnopyranosyl)]imberbic acid (108) [41].

In addition, $\beta$-D-glucopyranosyl $3 \beta, 19 \alpha$-dihydroxy-2oxo-urs-12-en-28-oate (76) was isolated from the leaves of Cameroonian $C$. bracteatum [32]; $\beta$-D-glucopyranosyl $2 \alpha, 3 \beta, 24$-trihydroxyolean-12-en-28-oate (77), bellericoside (78), $\beta$-D-glucopyranosyl $2 \alpha, 3 \beta, 6 \beta$-trihydroxyolean-12-en28-oate (79), asiatic acid (80), $\beta$-D-glucopyranosyl $2 \alpha, 3 \beta, 23,24$-tetrahydroxyurs-12-en-28-oate (81) from stems of C. laxum [33]; $\alpha$-amyrin (82), lupeol (83) from C. micranthum [34]; ursolic acid (115), corosolic acid (116), oleanolic acid (117), maslinic acid (118) from $C$. vendee and $C$. Zeyheri $[39,40]$; $3 \beta$-hydroxyolean-12-en-28-oic acid (107), 23$O$ - $[\alpha$-L-(4'-acetylrhamnopyranosyl)]imberbic acid (108), 23 acetoxy-3 $\beta$-acetylimberbic acid-29-methyl ester (109), 23-O[ $\alpha$-L-rhamnopyranosyl]-1,3 $\beta$-diacetylimberbic acid (110) from $C$. oliviforme [42]; $11 \alpha$-acetoxy-20,24-epoxy-25hydroxy-dammar-3-one (111), 20,24-epoxy-11 $\alpha, 25$ dihydroxy-dammar-3-one (112), 20,24-epoxy-12 $\beta, 25$ dihydroxy-dammar-3-one (113), arcapitin A (114) from $C$ nigricans [43]; $2 \alpha, 3 \beta$-dihydroxyurs-12-èn-28-oic acid (118) $6 \beta$-hydroxymaslinic acid (120) terminolic acid (121), methylsumaresinolate (122) were isolated from C. Zeyheri [44]; 3-oxo-cycloart-1,11,24-trien-23,21-olide (132) from $C$. erythrophyllum [47]; 3 $3,16 \alpha$-dihydroxy-13(17)mansumbinen-28-oic acid (133) from $C$. coccineum [48] and (16R)-16- $O$ - $\alpha$-L-arabinofuranosyl- $3 \beta$-hydroxydammara20,24-dièn-29-oic acid (134), (16R)-16-O- $\alpha$-L-arabinosyl$3 \beta$-hydroxymansumbin-13-en-28-oic acid (135) from $C$. rotundifolium [49].

\section{Phenolic Compounds}

\section{Flavonoids}

Flavonoids are also one of the major components of the genus Combretum. A total of 51 flavonoids belonging to the flavonols, flavanone, flavonones and chalcone structural groups (136-186) have been isolated and identified from Combretum species (Table 2).

Chemical investigation of the leaves of $C$. quadrangulare resulted in the isolation and structural determination of 9 flavonoids. These compounds are, kamatakenin (136), isokaempferide (137), 5,7,4'-trihydroxy-3,3'-dimethoxyflavone (138), 5,4'-dihydroxy-3,7,3'-trimethoxyflavone (139), (+)catechin (140) [25], (+)-gallocatechin (141), (-)-epicatechin (142), (-)-epigallocatechin (143), vitexin (144) [31,34], 5,7,3',5'-tetrahydroxy-3,4'-dimethoxyflavone (145), combretol (146), 3',5-dihydroxy-3,4',5,7-tetramethoxyflavone (147), 3',5-dihydroxy-3,4',5,7-tetramethoxyflavone (148), quercetin 3,4'-dimethyl ether (149), myricetin 3,3',4'trimethyl ether (150) [45].

C. micranthum was also reported to posses various and special types of flavonoid squeletons. Some of the identified compounds are, 3',4',5,7-tetrahydroxyflavan (151), 3',4',5',5,7-pentahydroxyflavan (152), isovitexin (153), orientin (154), homoorientin (155), myricetin-3-O-glucoside
(156), 2' '-O-galloylvotexin (157), 2' '-O-galloylisovotexin (158), 2', $O$-galloylorientin (159), 2' $-O$ galloylhomoorientin (160). New ring structures named piperidine-Flavan alkaloids were isolated from the $\mathrm{nBuOH}$ fraction of the leaves of $C$. micranthum. The 8 kinkeloids as they were named are, kinkeloid $A_{1}$ (161), kinkeloid $A_{2}$ (162), kinkeloid $\mathrm{B}_{1}(\mathbf{1 6 3})$, kinkeloid $\mathrm{B}_{2}(\mathbf{1 6 4})$, kinkeloid $\mathrm{C}_{1}(\mathbf{1 6 5})$, kinkeloid $C_{2}(\mathbf{1 6 6})$, kinkeloid $D_{1}(\mathbf{1 6 7})$, kinkeloid $D_{2}(\mathbf{1 6 8})$ [34].

Apigenin (169), genkwanin (170), 5-hydroxy-7,4'dimethoxyflavone (171), rhamnocitrin (172), kaempferol (173), quercetin-5,3'-dimethylether (174) and rhamnazin (175) was identified as flavonoid constituents from $C$. erythrophyllum [50]. Quercetin (178), 3-0-methylquercetin (179), quercetrin (180), 5,3', 4' -trihydroxy-3,7dimethoxyflavone (181) and 5,3'-dihydroxy-3,7,4'trimethoxyflavone (182) was also isolated from C. leprosum $[39,40]$; cardamonin (183) and pinocembrin (184) from $C$. apiculatum [51]; dillenetin (176) and isorhamnetin (177) from $C$. lanceolatum [52] and 2,4,4'-trihydroxychalcon (185) and eriodictyol (186) from C. yannanense [53].

\section{Diarylpropanes}

Nine 1,3-diarylpropanes, combrequinone A-C (187-189), combretol A-E (190-194) and 1-(2-methoxy-4hydroxyphenyl) 3-(3-hydroxy-4-methoxy phenyl)-propane (195) were isolated from the aerial parts of $C$. yunnanense $[53,54]$. Investigations of the methanolic extract of stem of C. griffithii led to the isolation and structural elucidation of five other diarylpropanes named, 1-(4-hydroxy-2,5dimethoxyphenyl)-3-(4-hydroxy-3-methoxyphenyl)propane (196), 1-(3-hydroxy-2,4-dimethoxyphenyl)-3-(4-hydroxy-3methoxyphenyl)propane (197), 1-(4,5-dihydroxy-2methoxyphenyl)-3-(3,4-dimethoxyphenyl)propane (198), 1(2-hydroxy-4-methoxyphenyl)-3-(4-hydroxy-3-

methoxyphenyl)propane (199) and 1-[2-(5-methoxy-1,4benzoquinone)])-3-(4-hydroxy-3-methoxyphenyl)propane (200) [55].

\section{Phenanthrenes and 9, 10-Dihydrophenanthrebnes}

Several phenanthrenes and 9, 10-dihydrophenanthrebnes have been isolated from various species of the genus Combretum. They are, 4,7-dihydroxy-2,3,6-trimethoxyphenanthrene (201), 2,6-dihydroxy-3,4,7-trimetho-xyphenanthrene (202), 4,6,7-trihydroxy-2,3-dimethoxyphen-anthrene (203), 2,3,7-dihydroxy-4,6-trimethoxyphenanthrene (204), 3,6,7dihydroxy-2,4-dimethoxyphenanthrene (205), 3,7dihydroxy-2,4,6-trimethoxyphenanthrene (206) from the heartwood of $C$. apiculatum; 7-hydroxy-2,4,6-trimethoxyphenanthrene (207), 7-hydroxy-2,3,4,6-tetramethoxyphenanthrene (208), 2,7-hydroxy-3,4,6-tetramethoxyphenanthrene (209), 2,6,7-trihydroxy-3,4-dimethoxyphenanthrene (210) from the heartwood of $C$. Psidioides and 3,6-dihydroxy-2,4,7-trimethoxyphenanthrene (211) from the heartwood of $C$. Hereroense for phenanthrenes and 2,6dihydroxy-3,4,7-trimethoxy-9,10-dihydrophenanthrene (212), 4,7-dihydroxy-2,6-dimethoxy-9,10-dihydrophenanthrene (213), 2,6-dihydroxy-4,7-dimethoxy-9,10-dihydrophenanthrene (214), 4,7-dihydroxy-2,3,6-trimethoxy-9,10dihydrophenanthrene (215), 2-hydroxy-3,4,6,7tetramethoxy-9,10-dihydrophenanthrene

(216), 


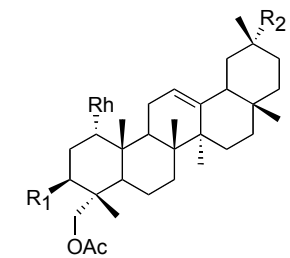

$104: \mathrm{R} 1=\mathrm{OAC} \mathrm{R} 2=\mathrm{COOH}$ 105: $\mathrm{R} 1=\mathrm{OH} \quad \mathrm{R} 2=\mathrm{COOCH} 3$ $106: \mathrm{R} 1=\mathrm{OAC} \quad \mathrm{R} 2=\mathrm{COOCH} 3$<smiles>CC1(C)CCC2(C(=O)O)CCC3C(=CCC4C3(C)CCC3C(C)(C)C(O)CCC34C)C2C1</smiles>

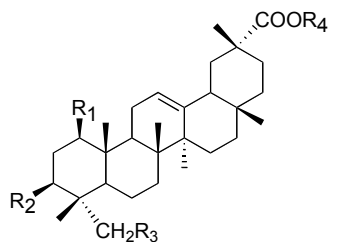

108: R1 $=\mathrm{OH} \quad \mathrm{R} 2=\mathrm{OH} \quad \mathrm{R} 3=4-\mathrm{Ac}-\mathrm{O}-\mathrm{Rh} \quad \mathrm{R} 4=\mathrm{H}$ 109: $\mathrm{R} 1=\mathrm{OH} \quad \mathrm{R} 2=\mathrm{Ac} \quad \mathrm{R} 3=\mathrm{OAC} \quad \mathrm{R} 4=\mathrm{Me}$ 110: $R 1=R 2=A c \quad R 3=-O-R h \quad R 4=H$

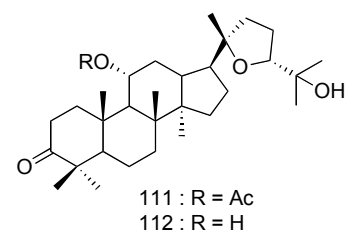

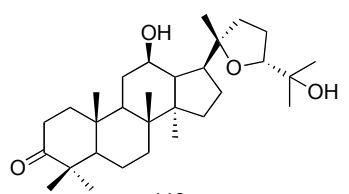

113

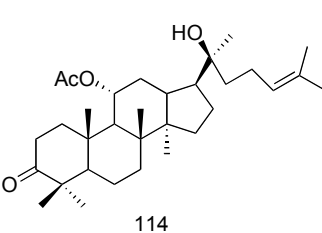

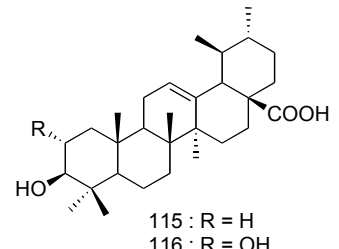

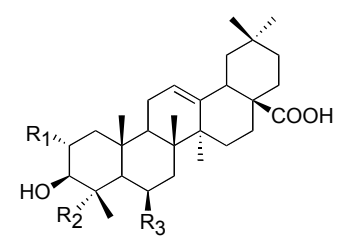

$117: R 1=H \quad R 2=M e$ $118: \mathrm{R} 1=\mathrm{OH} \mathrm{R} 2=\mathrm{Me}$ $119: \mathrm{R} 1=\mathrm{OH} \mathrm{R} 2=\mathrm{Me}$ $120: R 1=O H R 2=$ $121: \mathrm{R} 1=\mathrm{OH}$ R2 $=\mathrm{CH} 2 \mathrm{OH} \mathrm{R} 3=\mathrm{OH}$

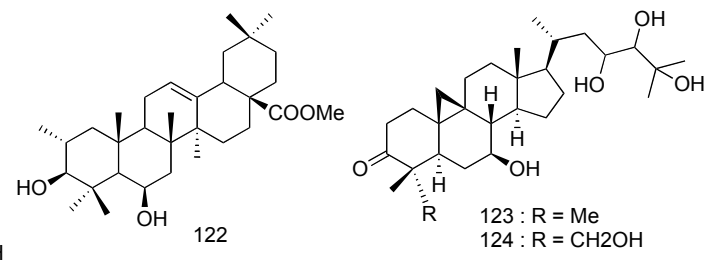

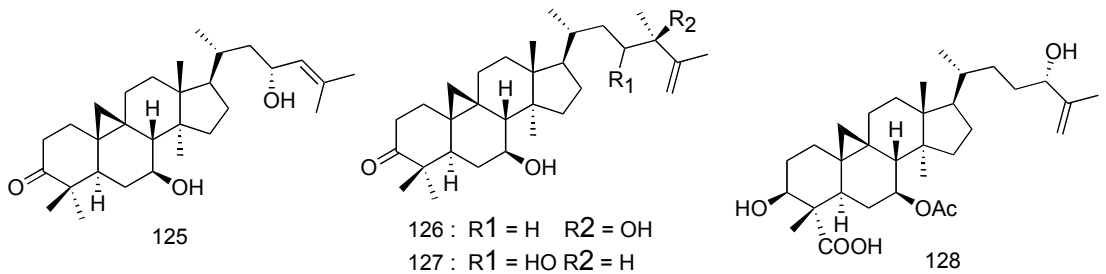

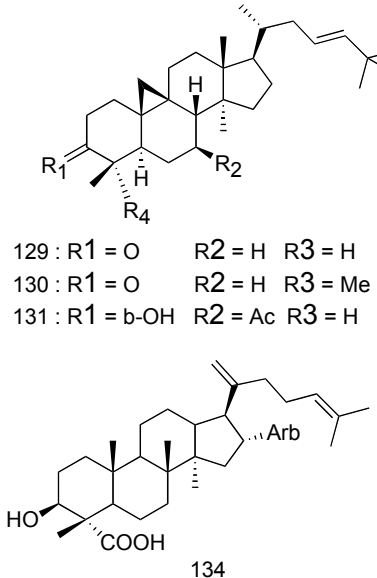
127: R1 = HOR2 = H 128 $\mathrm{OR}_{3}$

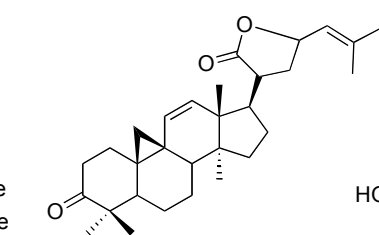

132

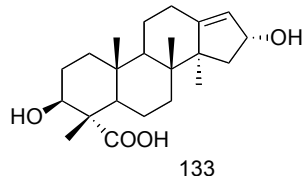

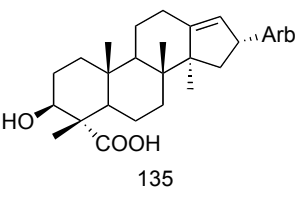


Table 2. Other Non-essential Oil Compounds Isolated and Identified from Combretum

\begin{tabular}{|c|c|c|c|}
\hline & Gallic acid and derivatives & & \\
\hline 239 & methyl gallate & $\begin{array}{l}\text { C. quadrangulare } \\
\text { C. erythrophyllum }\end{array}$ & {$[29,62]$} \\
\hline 240 & Ellagic acid and derivatives & & \\
\hline 241 & ellagic acid & C. yannanense & {$[66]$} \\
\hline 242 & 4-( $\alpha$-rhamnopyranosyl)ellagic acid & C. yannanense & {$[66]$} \\
\hline 243 & 4-(4"-O-acetyl- $\alpha$-rhamnopyranosyl)ellagic acid & C. yannanense & [66] \\
\hline 246 & 3,4,3'-tri- $O$-methylflavellagic acid-4'- $\beta$-D-glucoside & C. kraussii & {$[67]$} \\
\hline 247 & 2,3-(S)-hexahydroxydiphenoyl-D-glucose & C. glutinosum & {$[68]$} \\
\hline 248 & punicalin & C. glutinosum & [68] \\
\hline 249 & punicalagin & $\begin{array}{l}\text { C. molle } \\
\text { C. glutinosum }\end{array}$ & {$[20,68]$} \\
\hline \multirow[t]{2}{*}{250} & combreglutinin & C. glutinosum & {$[68]$} \\
\hline & Steroids & & \\
\hline 251 & $\beta$-sitosterol & $\begin{array}{l}\text { C. quadrangulare, } \\
\text { C. yannanense }\end{array}$ & {$[26,54]$} \\
\hline 255 & 5-methoxy-(-)-isolariciresinol & C. quadrangulare & [29] \\
\hline \multirow[t]{2}{*}{256} & 5-methoxy-9 $\beta$-xylopyranosyl-(-)-isolariciresinol & C. quadrangulare & [29] \\
\hline & Cyclobutanes & & \\
\hline 257 & $1 \alpha, 2 \beta$-di-(2,6-dimethoxy-4-hydroxybenzoyl)-( $3 \alpha, 4 \beta)$-diphenylcyclobutane & C. albopunctatum & [69] \\
\hline \multirow[t]{2}{*}{258} & $\begin{array}{l}1 \alpha \text {-(2-methoxy-4,6-dihydroxy-benzoyl)-2 } \beta \text {-(2,6-dimethoxy-4-hydroxybenzoyl)- } \\
(3 \alpha, 4 \beta) \text {-diphenylcyclobutane }\end{array}$ & C. albopunctatum & [69] \\
\hline & Lipids & & \\
\hline 259 & 1,2-di- $O$ - $\alpha$-linolenoyl-3-O- $\beta$ - $D$-galactopyranosyl-sn-glycerol & C. bracteatum & [32] \\
\hline \multirow[t]{2}{*}{260} & $\begin{array}{l}\text { 1,2-di- } O \text { - } \alpha \text {-linolenoyl-3- } O \text { - }[\alpha-D \text {-galactopyranosyl-(1 6)- } O-\beta \text { - } D \text {-galactopyranosyl]- } \\
s n \text {-glycerol }\end{array}$ & C. bracteatum & [32] \\
\hline & Coumarin & & \\
\hline 261 & scopoletin & C. yannanense & [54] \\
\hline
\end{tabular}

6,7-dihydroxy-2,3,4-trimethoxy-9,10-dihydrophenanthrene (217) and 4,6,7-trihydroxy-2,3-dimethoxy-9,10-dihydrophenanthrene (218) from C. apiculatum; 7-hydroxy-2,4,6trimethoxy-9,10-dihydrophenanthrene (219), 7-hydroxy- 2,3,4,6-tetramethoxy-9,10-dihydrophenanthrene (220), 2,7dihydroxy-3,4,6-trimethoxy-9,10-dihydrophenanthrene (221), 2,6,7-trihydroxy-3,4-dimethoxy-9,10-dihydrophenanthrene (222) C. Psidioides, 3,6-dihydroxy-2,4,7- 
<smiles>[R6]Oc1cc(O)c2c(c1)OC(c1ccc(O)c(O)c1)C(O[14C](=O)O)C2=O</smiles>

136: R1 = Me R2 = H

$137: \mathrm{R} 1=\mathrm{R} 2=\mathrm{H}$

$138: \mathrm{R} 1=\mathrm{H} \quad \mathrm{R} 2=\mathrm{Me}$

139: R1 = Me R2 $=\mathrm{Me}$

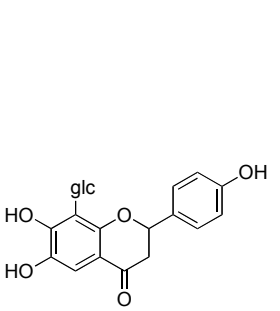

144<smiles>[R]c1cc(C2CCc3c(O)cc(O)cc3O2)cc(O)c1O</smiles>

$151: \mathrm{R}=\mathrm{H}$

$152: \mathrm{R}=\mathrm{OH}$

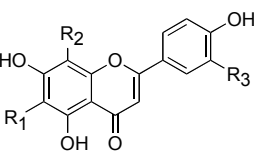

157: R1 = H R2 = O- galloyl-C-glc R3 = H 158: $R 1=0$ - galloyl-C-glc $R 2=R 3=H$ $159:$ R1 = H R2 = O- galloyl-C-glc R3 = $160: R 1=0$ - galloyl-C-glc $R 2=H \quad R 3=O H$

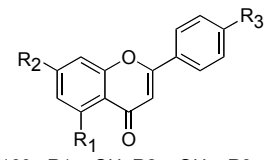

169: R1 $=\mathrm{OH} \quad \mathrm{R} 2=\mathrm{OH} \quad \mathrm{R} 3=\mathrm{OH}$ 170: $\mathrm{R} 1=\mathrm{OH} R 2=\mathrm{OMe} \mathrm{R} 3=\mathrm{OH}$ 171: R1 $=\mathrm{OH}$ R2 $=\mathrm{OMe} \mathrm{R} 3=\mathrm{OMe}$<smiles></smiles>

$176: \mathrm{R} 1=\mathrm{H} \quad \mathrm{R} 2=\mathrm{Me} \quad \mathrm{R} 3=\mathrm{Me}$ $177: R 1=H \quad R 2=M e R 3=H$ 178: R1 $=H \quad R 2=H \quad R 3=H$ 179: R1 = Me R2 = H R3 = H $180: R 1=R h R 2=H \quad R 3=H$<smiles>O=C1CC(c2ccccc2)Oc2cc(O)cc(O)c21</smiles><smiles>[R]c1cc(O)c(O)cc1[C@@H]1Oc2cc(O)cc(O)c2C[C@H]1O</smiles>

$140: \mathrm{R}=\mathrm{H}$ $141: \mathrm{R}=\mathrm{OH}$<smiles>O[R]1c2cc(O)c(O)cc2Oc2cc(O)cc(O)c21</smiles>

$142: \mathrm{R}=\mathrm{H}$ $143: \mathrm{R}=\mathrm{OH}$<smiles>[R3]c1cc([R2])c2c(=O)c([R2])c(-c3cc([R6])c([R5])c([R6])c3)oc2c1</smiles>

145: R1 $=\mathrm{OMe} \quad \mathrm{R} 2=\mathrm{OH} \quad \mathrm{R} 3=\mathrm{OH} \quad \mathrm{R} 4=\mathrm{OH} \quad \mathrm{R} 5=\mathrm{OMe} \quad \mathrm{R} 6=\mathrm{OH}$ 146: $\mathrm{R} 1=\mathrm{OMe} \quad \mathrm{R} 2=\mathrm{OH} \mathrm{R} 3=\mathrm{OMe} \mathrm{R} 4=\mathrm{OMe} \mathrm{R} 5=\mathrm{OMe} \mathrm{R} 6=\mathrm{OMe}$ 147: R1 = OMe R2 =OH R3 = OMe R4 =OH R5 =OMe R6 =OMe 148: R1 = OMe R2 =OH R3 = OMe R4 =OMe R5 =OH R6 $=\mathrm{H}$ 149: R1 = OMe $R 2=\mathrm{OH} \quad \mathrm{R} 3=\mathrm{OH} \quad \mathrm{R} 4=\mathrm{OH} \quad \mathrm{R} 5=\mathrm{OMe} \quad \mathrm{R} 6=\mathrm{H}$ $150: \mathrm{R} 1=\mathrm{OMe} \quad \mathrm{R} 2=\mathrm{OH} \mathrm{R} 3=\mathrm{OH} \quad \mathrm{R} 4=\mathrm{OMe} \mathrm{R} 5=\mathrm{OMe} \quad \mathrm{R} 6=\mathrm{OH}$<smiles>[R5]c1cc(-c2oc3c([R2])c(O)c([R])c(O)c3c(=O)c2[R3])cc([R4])c1O</smiles>

153: R1 = C-glc R2 = R3 = R4 = R5 = H $154: \mathrm{R} 1=\mathrm{R} 3=\mathrm{R} 5=\mathrm{H}$ R2 = C-glc R4 $=\mathrm{OH}$ 155: R1 = C-glc $\mathrm{R} 2=\mathrm{R} 3=\mathrm{R} 5=\mathrm{H} \quad \mathrm{R} 4=\mathrm{OH}$ $156: \mathrm{R} 1=\mathrm{R} 2=\mathrm{H}$ R3 $=$ O-glc $\mathrm{R} 4=\mathrm{R} 5=\mathrm{OH}$<smiles>[R1]c1cc([C@H]2Oc3c([R2])c(O)c([R1])c(O)c3C[C@H]2[R3])cc([R5])c1O</smiles>

161: R1 = 2-piperidinyl $R 2=R 3=R 4=R 5=H$ $162: R 2=2$-piperidinyl $R 1=R 3=R 4=R 5=H$ 163: $\mathrm{R} 1=$ 2-piperidinyl $\mathrm{R} 2=\mathrm{R} 3=\mathrm{R} 5=\mathrm{H} \quad \mathrm{R} 4=\mathrm{OH}$ 164: R2 = 2-piperidinyl $\mathrm{R} 1=\mathrm{R} 3=\mathrm{R} 5=\mathrm{H} \quad \mathrm{R} 4=\mathrm{OH}$ 165: R1 = 2-piperidinyl $\mathrm{R} 2=\mathrm{R} 3=\mathrm{H} \quad \mathrm{R} 4=\mathrm{R} 5=\mathrm{OH}$ 166: $\mathrm{R} 2=$ 2-piperidinyl $\mathrm{R} 1=\mathrm{R} 3=\mathrm{H} \quad \mathrm{R} 4=\mathrm{R} 5=\mathrm{OH}$ 167: R1 $=$ 2-piperidinyl $\mathrm{R} 2=\mathrm{H} \quad \mathrm{R} 3=\mathrm{R} 4=\mathrm{R} 5=\mathrm{OH}$ 168: R2 $=2$-piperidinyl $\mathrm{R} 1=\mathrm{H} \quad \mathrm{R} 3=\mathrm{R} 4=\mathrm{R} 5=\mathrm{OH}$<smiles>[R2]c1cc([R7])c2c(=O)cc(-c3ccc([R3])c([R4])c3)oc2c1</smiles>

172: R1 $=\mathrm{OH}$ R2 $=\mathrm{OMe} \mathrm{R} 3=\mathrm{OH} \mathrm{R} 4=\mathrm{H}$ 173: R1 $=\mathrm{OH} \quad \mathrm{R} 2=\mathrm{OH} \quad \mathrm{R} 3=\mathrm{OH} \mathrm{R} 4=\mathrm{H}$ $174: \mathrm{R} 1=\mathrm{OMe} \mathrm{R} 2=\mathrm{OH} \quad \mathrm{R} 3=\mathrm{OH} \mathrm{R} 4=\mathrm{OMe}$ $175: \mathrm{R} 1=\mathrm{OH} \quad \mathrm{R} 2=\mathrm{OMe} \mathrm{R} 3=\mathrm{OH} \mathrm{R} 4=\mathrm{OMe}$<smiles></smiles>
181: R1 = Me R2 = Me R3 = H $182: \mathrm{R} 1=\mathrm{R} 2=\mathrm{R} 3=\mathrm{Me}$

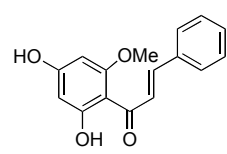

183<smiles>O=C(C(O)=Cc1ccc(O)cc1)c1ccccc1O</smiles><smiles>CC(C)(C)C</smiles> 

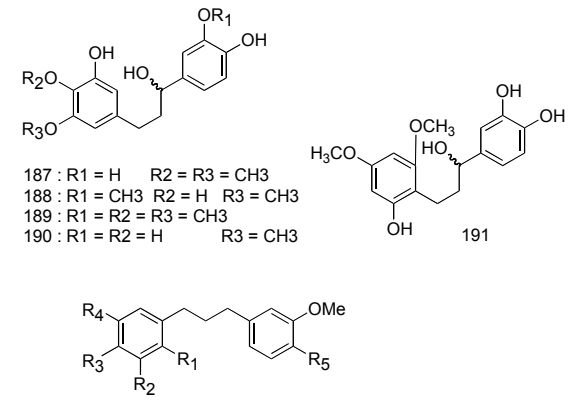

192: R1 = OMe R2 = H R3 $=\mathrm{OH} \quad \mathrm{R} 4=\mathrm{OMe} \mathrm{R} 5=\mathrm{OH}$ 193: R1 = OMe R2 $=\mathrm{OH} R 3=\mathrm{OMe} R 4=\mathrm{H} \quad \mathrm{R} 5=\mathrm{OH}$ $194: \mathrm{R} 1=\mathrm{OMe} \mathrm{R} 2=\mathrm{H} \quad \mathrm{R} 3=\mathrm{OH} \quad \mathrm{R} 4=\mathrm{OH} \quad \mathrm{R} 5=\mathrm{OMe}$ 195: R1 =OH $\quad \mathrm{R} 2=\mathrm{H} \quad \mathrm{R} 3=\mathrm{OMe} \quad \mathrm{R} 4=\mathrm{H} \quad \mathrm{R} 5=\mathrm{OH}$

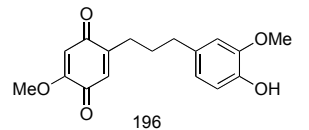

196

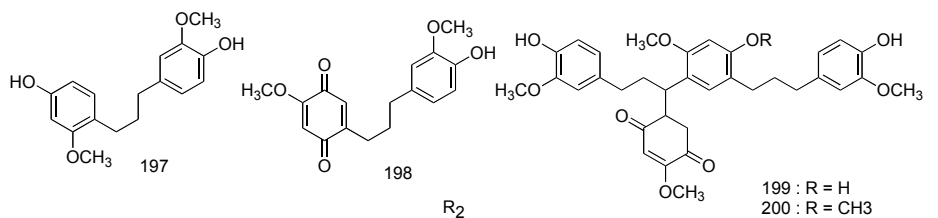

$199: \mathrm{R}=\mathrm{H}$
$200: \mathrm{R}=\mathrm{CH} 3$

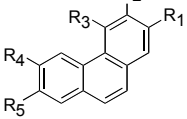

201: $\mathrm{R} 1=\mathrm{OMe} \quad \mathrm{R} 2=\mathrm{OMe} \quad \mathrm{R} 3=\mathrm{OH} \quad \mathrm{R} 4=\mathrm{OMe} \quad \mathrm{R} 5=\mathrm{OH}$ 202: $\mathrm{R} 1=\mathrm{OH} \quad \mathrm{R} 2=\mathrm{OMe} \quad \mathrm{R} 3=\mathrm{OMe} \quad \mathrm{R} 4=\mathrm{OH} \quad \mathrm{R} 5=\mathrm{OMe}$ 203: R1 $=\mathrm{OMe} \quad \mathrm{R} 2=\mathrm{OMe} \quad \mathrm{R} 3=\mathrm{OH} \quad \mathrm{R} 4=\mathrm{OH} \quad \mathrm{R} 5=\mathrm{OH}$ 204: $\mathrm{R} 1=\mathrm{OH} \quad \mathrm{R} 2=\mathrm{OH} \quad \mathrm{R} 3=\mathrm{OMe} \quad \mathrm{R} 4=\mathrm{OMe} \mathrm{R} 5=\mathrm{OH}$

205: $\mathrm{R} 1=\mathrm{OMe} \quad \mathrm{R} 2=\mathrm{OH} \quad \mathrm{R} 3=\mathrm{OMe} \quad \mathrm{R} 4=\mathrm{OH} \quad \mathrm{R} 5=\mathrm{OH}$ 206: $\mathrm{R} 1=\mathrm{OMe} \quad \mathrm{R} 2=\mathrm{OH} \quad \mathrm{R} 3=\mathrm{OMe} \quad \mathrm{R} 4=\mathrm{OMe} \mathrm{R} 5=\mathrm{OH}$ $207: \mathrm{R} 1=\mathrm{OMe} \quad \mathrm{R} 2=\mathrm{H} \quad \mathrm{R} 3=\mathrm{OMe} \quad \mathrm{R} 4=\mathrm{OMe} \quad \mathrm{R} 5=\mathrm{OH}$ 208: R1 $=\mathrm{OMe} \quad \mathrm{R} 2=\mathrm{OMe} \quad \mathrm{R} 3=\mathrm{OMe} \quad \mathrm{R} 4=\mathrm{OH} \quad \mathrm{R} 5=\mathrm{OH}$ 209: $\mathrm{R} 1=\mathrm{OH} \quad \mathrm{R} 2=\mathrm{OMe} \quad \mathrm{R} 3=\mathrm{OMe} \quad \mathrm{R} 4=\mathrm{OMe} \mathrm{R} 5=\mathrm{OH}$ 210: $\mathrm{R} 1=\mathrm{OH} \quad \mathrm{R} 2=\mathrm{OMe} \quad \mathrm{R} 3=\mathrm{OMe} \quad \mathrm{R} 4=\mathrm{OH} \quad \mathrm{R} 5=\mathrm{OH}$ 211: $\mathrm{R} 1=\mathrm{OMe} \quad \mathrm{R} 2=\mathrm{OH} \quad \mathrm{R} 3=\mathrm{OMe} \quad \mathrm{R} 4=\mathrm{OH} \quad \mathrm{R} 5=\mathrm{OMe}$

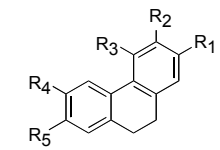

213: $\mathrm{R} 1=\mathrm{OH} \quad \mathrm{R} 2=\mathrm{OMe} \quad \mathrm{R} 3=\mathrm{OMe} \quad \mathrm{R} 4=\mathrm{OH} \quad \mathrm{R} 5=\mathrm{OMe}$ $214: \mathrm{R} 1=\mathrm{OH} \quad \mathrm{R} 2=\mathrm{H} \quad \mathrm{R} 3=\mathrm{OMe} \quad \mathrm{R} 4=\mathrm{OH} \quad \mathrm{R} 5=\mathrm{OMe}$ 215: $\mathrm{R} 1=\mathrm{OMe} \mathrm{R} 2=\mathrm{OMe} \quad \mathrm{R} 3=\mathrm{OH} \quad \mathrm{R} 4=\mathrm{OMe} \mathrm{R} 5=\mathrm{OH}$ 216: $\mathrm{R} 1=\mathrm{OMe} \mathrm{R} 2=\mathrm{OMe} \quad \mathrm{R} 3=\mathrm{OH} \quad \mathrm{R} 4=\mathrm{OH} \quad \mathrm{R} 5=\mathrm{OH}$ $217: \mathrm{R} 1=\mathrm{OMe} R 2=\mathrm{OMe} \quad \mathrm{R} 3=\mathrm{OMe} \quad \mathrm{R} 4=\mathrm{OH} \quad \mathrm{R} 5=\mathrm{OH}$ 218: $\mathrm{R} 1=\mathrm{OH} \quad \mathrm{R} 2=\mathrm{OMe} \quad \mathrm{R} 3=\mathrm{OMe} \mathrm{R} 4=\mathrm{OMe} \mathrm{R} 5=\mathrm{OMe}$ 219: R1 $=$ OMe R2 $=\mathrm{H} \quad \mathrm{R} 3=\mathrm{OMe} \quad \mathrm{R} 4=\mathrm{OMe} \mathrm{R} 5=\mathrm{OH}$ 220: $\mathrm{R} 1=\mathrm{OMe} \mathrm{R} 2=\mathrm{OMe} \quad \mathrm{R} 3=\mathrm{OMe} \mathrm{R} 4=\mathrm{OMe} \mathrm{R} 5=\mathrm{OH}$ 221: $\mathrm{R} 1=\mathrm{OH} \quad \mathrm{R} 2=\mathrm{OMe} \quad \mathrm{R} 3=\mathrm{OMe} \quad \mathrm{R} 4=\mathrm{OMe} \mathrm{R} 5=\mathrm{OH}$ $222: \mathrm{R} 1=\mathrm{OH} \quad \mathrm{R} 2=\mathrm{OMe} \quad \mathrm{R} 3=\mathrm{OMe} \quad \mathrm{R} 4=\mathrm{OH} \quad \mathrm{R} 5=\mathrm{OH}$ 223: R1 = OMe R2 =OH $\quad \mathrm{R} 3=\mathrm{OMe} \quad \mathrm{R} 4=\mathrm{OH} \quad \mathrm{R} 5=\mathrm{OMe}$
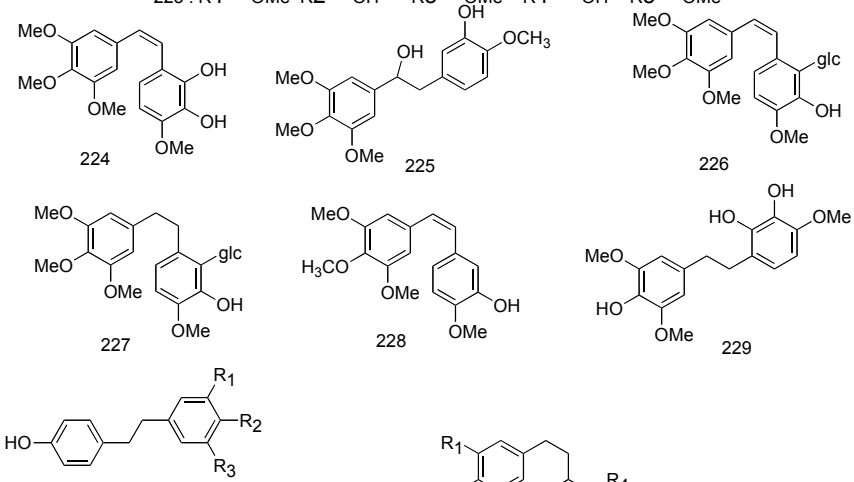

$230: \mathrm{R} 1=\mathrm{OMe} \mathrm{R} 2=\mathrm{OMe} \mathrm{R} 3=\mathrm{OH}$ $231: \mathrm{R} 1=\mathrm{OMe} \mathrm{R} 2=\mathrm{OMe} \mathrm{R} 3=\mathrm{OMe}$ $232: R 1=O M e R 2=O H \quad R 3=O M e$

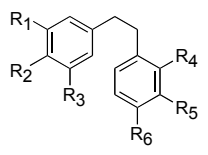

233: $\mathrm{R} 1=\mathrm{OMe} \quad \mathrm{R} 2=\mathrm{H} \quad \mathrm{R} 3=\mathrm{H} \quad \mathrm{R} 4=\mathrm{H} \quad \mathrm{R} 5=\mathrm{OH} \quad \mathrm{R} 6=\mathrm{OM}$ 234: $\mathrm{R} 1=\mathrm{OMe} \quad \mathrm{R} 2=\mathrm{OMe} \quad \mathrm{R} 3=\mathrm{OMe} \quad \mathrm{R} 4=\mathrm{H} \quad \mathrm{R} 5=\mathrm{H} \quad \mathrm{R} 6=\mathrm{OH}$ 235: R1 = OMe $\quad \mathrm{R} 2=\mathrm{OMe} \quad \mathrm{R} 3=\mathrm{OH} \quad \mathrm{R} 4=\mathrm{H} \quad \mathrm{R} 5=\mathrm{H} \quad \mathrm{R} 6=\mathrm{OH}$ $236: \mathrm{R} 1=\mathrm{OMe} \quad \mathrm{R} 2=\mathrm{OH} \quad \mathrm{R} 3=\mathrm{OMe} \quad \mathrm{R} 4=\mathrm{H} \quad \mathrm{R} 5=\mathrm{H} \quad \mathrm{R} 6=\mathrm{OH}$ 237: R1 = OMe R2 = OMe R3 = OMe R4 = H R5 = OH R6 $=\mathrm{H}$ 
<smiles>O=C(O)c1cc(O)c(O)c(O)c1</smiles>

$238: \mathrm{R}=\mathrm{H}$ $239: \mathrm{R}=\mathrm{Me}$

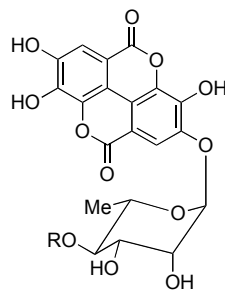

$24: R=H$ $243: R=A C$

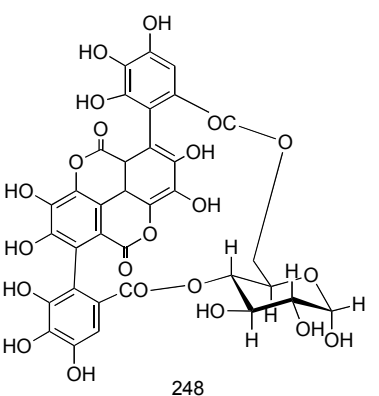

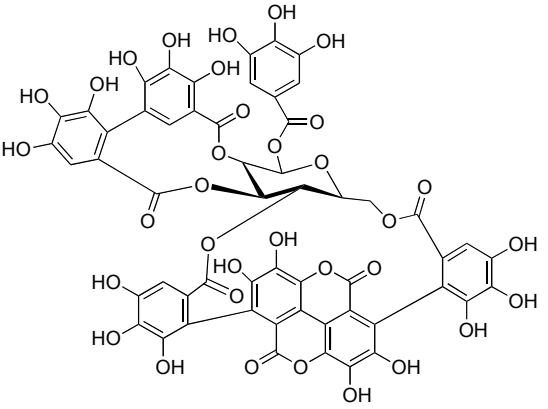

250

240

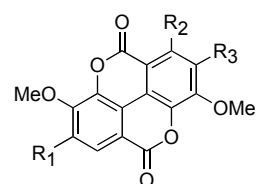

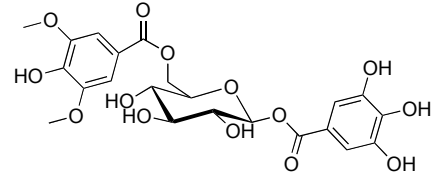

244: R1 $=\mathrm{OMe} \mathrm{R} 2=\mathrm{OH} \mathrm{R} 3=\mathrm{OH}$ 245: R1 $=\mathrm{OH} \quad \mathrm{R} 2=\mathrm{H} \quad \mathrm{R} 3=\mathrm{OH}$

246: $\mathrm{R} 1=\mathrm{OMe} \mathrm{R} 2=\mathrm{OH} \mathrm{R} 3=\mathrm{glc}$
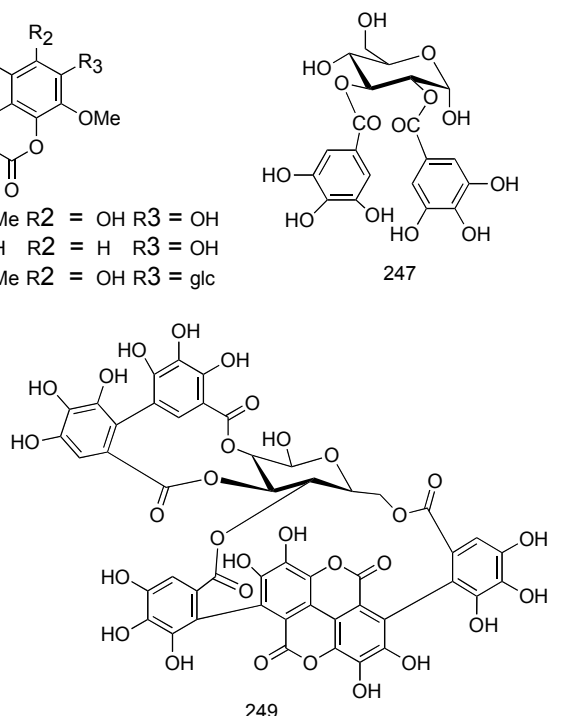

249

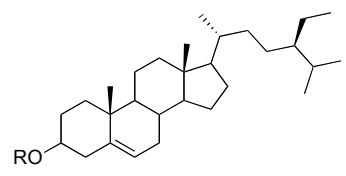

$251: R=H$
$252: R=$ glc
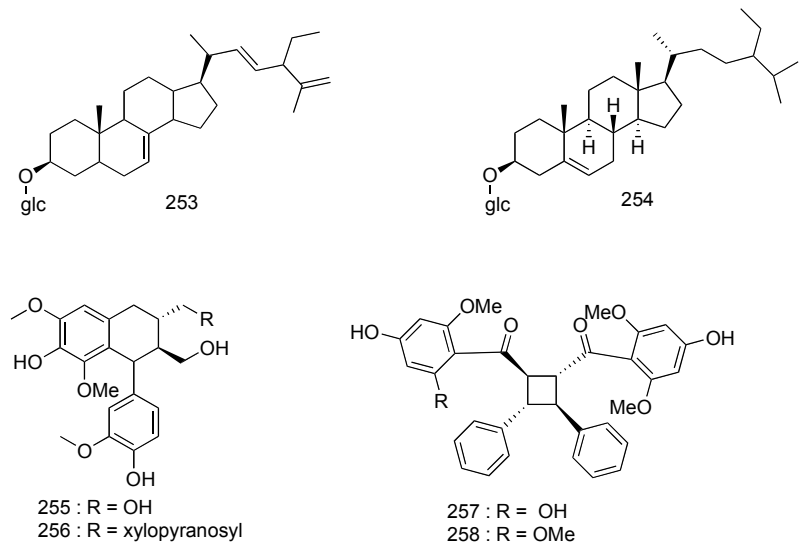

$257: \mathrm{R}=\mathrm{OH}$

$258: \mathrm{R}=\mathrm{OMe}$

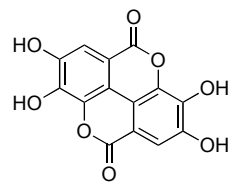

241 
trimetho-xy-9,10-dihydrophenanthrene (223) from C. Hereroense for 9, 10-dihydrophenanthrebnes [56-60].

\section{Stilbenes and Dihydrostilbenes}

Six stilbenes and eight dihydrostilbenes have been isolated and identified from the Combretum species. These compounds include combretastatin A-1(224), (-)combretastatin (225), combretastatin A-1,2'- $\beta$-D-glucoside (226), combretastatin B-1,2'- $\beta$-D-glucoside (227) from $C$. erythrophyllum; combretastatin A-4 (228) from the stem wood of C. Cafrum; combretastatin B-5 (229) from the leaves of South African C. woodii; 3,4'-dihydroxy-4,5dimethoxybibenzyl (230) from C. molle; 4'-hydroxy-3,4,5trimethoxybibenzyl (231) and 4,4'-dihydroxy-3,5dimethoxybibenzyl (232) from C. Psidioides and, 3hydroxy-2,3'methoxybibenzyl (233), 2'-hydroxy-3,4,5trimethoxybibenzyl (234), 2,3'-dihydroxy-4',5'dimethoxybibenzyl (235), 2,4'-dihydroxy-3,5dimethoxybibenzyl (236) and 3'-hydroxy-3,4,5trimethoxybibenzyl (237) from the leaves and stem barks of C. apiculatum [56, 58, 60-64].

\section{Others Compounds}

Gallic acid (238), methyl gallate (239) and 1-O-galloyl6- $O$-(4-hydroxy-3,5-dimethoxy)benzoyl- $\beta$-D-glucose (240), was yielded from $C$. quadrangulare [ 29,65]. Ellagic acid derivatives were also identified from Combretum species. These include, ellagic acid (241), 4- $(\alpha-$ rhamnopyranosyl)ellagic acid (242) and 4-(4"'-O-acetyl- $\alpha$ rhamnopyranosyl)ellagic acid (243) from the branches of Chinese C. yannanense; 3,3', 4'-tri-O-methylflavellagic acid (244), 3,3'-di- $O$-methylellagic acid (245) and 3,4,3'-tri- $O$ methylflavellagic acid-4'- $\beta$-D-glucoside (246) from the leaves of C. kraussii and 2,3-(S)-hexahydroxydiphenoyl-Dglucose (247) punicalin (248), punicalagin (249) and combreglutinin (250) from the leaves and stem barks of C. glutinosum [65-68].

Four steroids were found from Combretum species. These steroids are, $\beta$-sitosterol (251) and $\beta$-sitosterol glucoside (252) from $C$. quadrangulare [26, 29]; 24-ethylcholesta7,22,25-triene-3-O- $\beta$-D-glucopyranoside (253) from C. $p a$ doides [38] and D-glucopyranoside-3 $\beta$-stigmast-5-en-3-yl (254) from C. leprosum [40].

In addition, 5-methoxy-(-)-isolariciresinol (255) and 5methoxy-9 $\beta$-xylopyranosyl-(-)-isolariciresinol (256) were isolated and identified from $C$. quadrangulare [29]; $1 \alpha, 2 \beta$ -

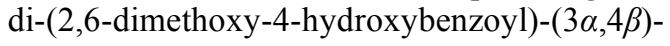

diphenylcyclobutane (257) and 1 $\alpha$-(2-methoxy-4,6dihydroxy-benzoyl)-2 $\beta$-(2,6-dimethoxy-4-hydroxybenzoyl)$(3 \alpha, 4 \beta)$-diphenylcyclobutane from (258) $C$. albopunctatum [69]; 1,2-di- $O$ - $\alpha$-linolenoyl-3- $O-\beta$ - $D$-galactopyranosyl-snglycerol (259) and 1,2-di-O- $\alpha$-linolenoyl-3-O-[ $\alpha-D-$ galactopyranosyl-(1 6)-O- $\beta$ - $D$-galactopyranosyl]-sn-glycerol (260) from $C$. bracteatum [32] and lastly scopoletin (261) from C. yannanense [54].

\section{CONCLUSION}

In different parts of Asia and Africa, Combretum species are widely used in folk medicine for the treatment of hepatitis, malaria, respiratory infections, and cancer. Phytochemi- cal investigations on 31 species of this genus led to the isolation of 261 components including 135 triterpenoids, 51 flavonoids, 14 diarylpropanes, 23 phenanthenes and derivatives, 14 stilbenoids and derivatives etc. On the basis of the above investigation, it is evident that $C$. quadrangulare is the most investigated specie and triterpenoids being the major constituents of the genus. Future studies should be directed on phytochemical investigation of those species with limited scientific literature.

\section{CONFLICT OF INTEREST}

The author(s) confirm that this article content has no conflicts of interest.

\section{ACKNOWLEDGEMENT}

Authors are thankful for the University of Karachi for the reception of several articles.

\section{REFERENCES}

[1] Hutching, A.; Scott, A.H.; Lewis, G.; Cunningham, A.B. Zulu Medicinal Plants-An Inventory; University of Natal Press, Pietermaritzburg, South Africa, 1996, pp. 27-41.

[2] Eloff, J.N.; Katerere, D.R.; McGaw, L.J. The biological activity and chemistry of the southern African Combretaceae. Journal of Ethnopharmacol., 2008, 119, 686-699.

[3] Wickens, G.E. Flora of Tropical East Africa. Combretaceae. East African community, 1973, p. 99.

[4] Watt, J.M.; Breyer-Brandwijk, M. G. The Medicinal and Poisonous Plants of Southern and Eastern Africa. E. and S. Livingstone Ltd, London, 1962, p. 194.

[5] Van-Wyk, B.; Van-Wyk, P. Field Guide to trees of Southern Africa; Struik Publishers, Cape Town, 1997, 536.

[6] Rogers, C.B.; Verotta, L. Chemistry and biological properties of the African Combretaceae. In: Hostettman K, Chinyanganga F, Maillard M, Wolfender, J-L., (Eds.). Chemistry, biological and pharmacological properties of African medicinal plants. Zimbabwe, Harare: University of Zimbabwe Publications, 1996, pp. 231-234.

[7] De Morais Lima, G.R.; Praxedes de Sales, I.R.; Dutra Caldas Filho, M.R.; Taveira de Jesus, N.Z.; De Sousa Falcão, H.; BarbosaFilho, J.M.; Silveira Cabral, A.G.; Lopes Souto, A.; Tavares, J.F.; Batista, L.M. Bioactivities of the Genus Combretum (Combretaceae): A Review. Molecules, 2012, 17, 9142-9206.

[8] Masoko, P.; Picard, J.; Eloff, J.N. The antifungal activity of twenty-four Southern African Combretum species (Combretaceae). South Afr. J. Bot., 2007, 73, 173-183.

[9] Ferrea, G.; Canessa, A.; Sampietro, F.; Cruciani, M.; Romussi, G.; Bassetti, D. In vitro activity of a Combretum micranthum extract against Herpes simplex virus types 1 and 2. Antiviral Res., 1993, 21, 317-325.

[10] Benoit, F.; Valentin, A.; Pelissier, Y.; Diafouka, F.; Marion, C.; Kone-Bamba, D.; Kone, M.; Mallie, M.; Yapo, A.; Bastide, J.M. In vitro antimalarial activity of vegetal extracts used in west african traditional medicine. Am. J. Trop. Med. Hyg., 1996, 54, 67-71.

[11] Chika, A.; Bello, S.O. Antihyperglycaemic activity of aqueous leaf extract of Combretum micranthum (Combretaceae) in normal and alloxan-induced diabetic rats. J. Ethnopharmacol., 2010, 129(1), 34-37.

[12] Ares, K; Mazumder, A; Bucar, F. Antibacterial and antifungal activities of extracts of Combretum molle. Ethiop. Med. J., 2006, 44(3), 269-277.

[13] Simon, M.K.; Ajanusi, O.J.; Abubakar, M.S.; Idris, A.L.; Suleiman, M.M. The anthelmintic effect of aqueous methanol extract of Combretum molle (R. Br. x. G. Don) (Combretaceae) in lambs experimentally infected with Haemonchus contortus. Veterinary Parasitol, 2012, 187(1-2), 280-284.

[14] Yeo, D.; N'Guessan, J.D.; Sea, T.; Coulibaly, Y.A.; Djaman, A.J.; Tako, N.A.; Yavo, J.C.; Guede-Guina, F. Evaluation de l'activité antiasthmatique et antitussive de Combretum molle, plante médici- 
nale de la pharmacopée ivoirienne. Phytothérapie, 2008, 6(6), 348351.

[15] Martini, N.; Eloff, J.N. The preliminary isolation of several antibacterial compounds from Combretum erythrophyllum (Combretaceae). J. Ethnopharmacol., 1998, 62, 255-263.

[16] Eloff, J.N. It is possible to use herbarium specimens to screen for antibacterial components in some plants. J. Ethnopharmacol., 1999, 67, 355-360.

[17] Bahar, A.; Tawfeq, A.A.H.; Passreiter, C.M.; Jaber, S.M. Combretene $\mathrm{A}$ and $\mathrm{B}$, Two new triterpenes from Combretum molle. Pharm. Biol., 2004, 42 (2), 109-113.

[18] Kemvoufo, P.B.; Barboni, L.; Teponno, R.B.; Mbiantcha, M.; Nguelefack, T.B.; Hee-Juhn, P.; Kyung-Tae, L.; Tapondjou, L.A. Polyhydroxyoleanane-type triterpenoids from Combretum molle and their anti-inflammatory activity. Phytochem. Lett., 2008, 1(4), 183-187.

[19] Jossang, A.; Seuleiman, M.; Maidou, E.; Bodo, B. Pentacyclic triterpenes from Combretum nigricans. Phytochemistry, 1996, 41(2), 591-594.

[20] Asres, K.; Bucar, F.; Knauder, E.; Yardley, V.; Kendrick, H.; Croft, S.L. In vitro antiprotozoal activity of extract and compounds from the stem bark of Combretum molle. Phytother. Res., 2001, 15(7), 613-617.

[21] Pegel, K.H.; Rogers, C.B. The characterisation of mollic acid 3 $\beta-$ $\mathrm{D}$-xyloside and its genuine aglycone mollic acid, two novel $1 \alpha$ hydroxycycloartenoids from Combretum molle. J. Chem. Soc., Perkin Trans., 1985, 1, 1711-1715.

[22] Rogers, C.B., Coombes, P.H. Mollic acid and its glycosides in the trichome secretions of Combretum petrophilum. Biochem. Syst. Ecol., 2001, 29(3), 329-330.

[23] Rogers, C.B. Isolation of the $1 \alpha$-hydroxycycloartenoid, mollic acid $\alpha$-l-arabinoside from Combretum edwardsii leaves. Phytochemistry, 1989, 28(1), 279-280.

[24] Ganzera, M.; Ellmerer-Müller, E.P.; Stuppner, H. Cycloartane triterpenes from Combretum quadrangulare. Phytochemistry, 1998, 49(3), 835-838.

[25] Banskota, A.H.; Tezuka, Y.; Tran, K.Q.; Tanaka, K.; Saiki, I.; Kadota, S. Thirteen novel cycloartane-type triterpenes from Combretum quadrangulare. J. Nat. Prod., 2000, 63(1), 57-64.

[26] Banskota, A.H.; Tezuka, Y.; Tran, K.Q.; Tanaka, K.; Saiki, I.; Kadota, S. Methyl quadrangularates A-D and related triterpenes from Combretum quadrangulare. Chem. Pharm Bull., 2000, 48(4), 496-504.

[27] Osborne, R.; Pegel, K.H. Jessic acid and related acid triterpenoids from Combretum elaeagnoides. Phytochemistry, 1984, 23, 635637.

[28] Adnyana, I.K.; Tezuka, Y.; Banskota, A.H.; Tran, K.Q.; Kadota, S. Three new triterpenes from the seeds of Combretum quadrangulare and their hepatoprotective activity. J. Nat. Prod., 2001, 64(3), 360363.

[29] Adnyana, I.K.; Tezuka, Y.; Banskota, A.H.; Xiong, Q.; Tran, K.Q.; Kadota, S. Quadranosides I-V, new triterpene glucosides from the seeds of Combretum quadrangulare. J. Nat. Prod., 2000, 63(4), 496-500.

[30] Masoko, P.; Mdee, L.K.; Mampuru, L.J.; Eloff, J.N. Biological activity of two related triterpenes isolated from Combretum nelsonii(Combretaceae) leaves. Nat. Prod. Res., 2008, 22(12), 10741084.

[31] Adnyana, I.K.; Tezuka, Y.; Awale, S.; Banskota, A.H.; Tran, K.Q.; Kadota, S. Quadranosides VI-XI, six new triterpene glucosides from the seeds of Combretum quadrangulare. Chem. Pharm. Bull., 2000, 48(8), 1114-1120.

[32] Ntchatcho, G.; Verotta, L.; Finzi, P.V.; Zanoni, G.; Vidari, G. A new beta-D-glucopyranosyl 2-oxo-urs-12-en-28-oate from the Cameroonian plant Combretum bracteatum. Nat. Prod. Commun., 2009, 4(12), 1631-1636.

[33] Bisoli, E.; Garcez, W.S.; Hamerski, L.; Tieppo, C.; Garcez, F.R. Bioactive pentacyclic triterpenes from the stems of Combretum laxum. Molecules, 2008, 13(11), 2717-2728.

[34] Welch, C.R. Chemistry and pharmacology of kinkéliba (Combretum micranthum), a West African medicinal plant; PhD Thesis, The State University of New Jersey, 2010, pp. 10-62.

[35] Rogers, C.B.; Subramony, G. The structure of imberbic acid, A $1 \alpha$-hydroxy pentacyclic triterpenoid from Combretum imberbe. Phytochemistry, 1988, 27(2), 531-533.
[36] Angeh, J.E.; Huang, X.; Sattler, I.; Swan, G.E.; Dahse, H.; Härtl, A.; Eloff, J.N. Antimicrobial and anti-inflammatory activity of four known and one new triterpenoid from Combretum imberbe (Combretaceae). J. Ethnopharmacol., 2007, 110(1), 56-60.

[37] Katerere, D.R.; Gray, A.I.; Nash, R.J.; Waigh, R.D. Antimicrobial activity of pentacyclic triterpenes isolated from African Combretaceae. Phytochemistry, 2003, 63, 81-88.

[38] Angeh, J.E.; Huang, X.; Swan, G.E.; Ute, M.; Sattler, I.; Eloff, J.N. Novel antibacterial triterpenoid from Combretum padoides (Combretaceae). Arkivoc, 2007, 9, 113-120.

[39] Facundo, V.A.; Andrade, C.H.S; Edilberto, R.S.; Braz-Filho, R.; Hufford, C.D. Triterpenes and flavonoids from Combretum leprosum. Phytochemistry, 1993, 32(2), 411-415.

[40] Facundo, V.A.; Katiuscia, A.R.; Moreira, L.S.; Sancho, J.L.; Teixeira, M.; Guerino, S.R.; Braz-Filho, R.; Edilberto, R.S. Two new cycloartanes from Combretum leprosum Mart. (Combretaceae). Rev. Latinoamer. Quim, 2008, 36(3), 76-82.

[41] Litaudon, M.; Jolly, C.; Le Callonec, C.; Cuong, D.D.; Retailleau, P.; Nosjean, O.; Nguyen, V.H.; Pfeiffer, B.; Boutin, J.A.; Guéritte, F. Cytotoxic pentacyclic triterpenoids from Combretum sundaicum and Lantana camara as inhibitors of Bcl-xL/BakBH3 domain peptide interaction. J. Nat. Prod., 2009, 72(7), 1314-1320.

[42] Wu, X.P.; Han, C.R.; Chen, G.Y.; Yuan, Y.; Xie, J.Y. Cytotoxic pentacyclic triterpenoids from Combretum oliviforme. Nat. Prod. Commun., 2010, 5(7), 1027-1030.

[43] Simon, G.; Dewelle, J.; Nacoulma, O.; Guissou, P.; Kiss, R.; Daloze, D.; Braekman, J.C. Cytotoxic pentacyclic triterpenes from Combretum nigricans. Fitoterapia, 2003, 74(4), 339-344.

[44] Runyoro, D.K.B.; Srivastava, S.K.; Darokar, M.P.; Olipa, N.D.; Cosam, C. J.; Mecky, I.N.M. Anticandidiasis agents from a Tanzanian plant, Combretum zeyheri. Med. Chem. Res., 2013, 22(3), 1258-1262.

[45] Toume, K.; Nakazawa, T.; Ohtsuki, T.; Arai, M.A.; Koyano, T. ; Kowithayakorn, T.; Ishibashi, M. Cycloartane triterpenes isolated from Combretum quadrangulare in a Screening Program for Death-Receptor expression enhancing activity. J. Nat. Prod., 2011, 74(2), 249-255.

[46] Aroke, A.S. Biological activities of extracts and isolated compounds from Bauhinia galpinii (Fabaceae) and Combretum vendae (Combretaceae) as potential antidiarrhoeal agents. PhD Thesis, Department of paraclinical sciences, Faculty of veterinary, University of Pretotia, South Africa, 2012, pp. 129-131.

[47] Rogers, C.B. Cycloartenoid dienone acids and lactones from Combretum erythrophyllum. Phytochemistry, 1998, 49(7), 2069-2076.

[48] Rasheduzzaman, C.; Norin, I. A hydroxylated mansumbinen-28-oic acid from Combretum coccineum. Biochem. Syst. Ecol., 2004, 32(4), 443-445.

[49] Rogers, C.B. Acidic dammarane arabinofuranosides from Combretum rotundifolium. Phytochemistry, 1995, 40(3), 833-836.

[50] Martini, N.D.; Katerere, D.R.P; Eloff, J.N. Biological activity of five antibacterial flavonoids from Combretum erythrophyllum (Combretaceae). J. Ethnopharmacol., 2004, 93(2-3), 207-212.

[51] Aderogba, M.A.; Kgatle, D.T.; McGaw, L.J.; Eloff, J.N. Isolation of antioxidant constituents from Combretum apiculatum subsp. Apiculatum. S. Afr. J. Botany, 2012, 79, 125-131.

[52] Araujo, L.C.J.; da Silva, V.C.; Dall'Oglio, E.L.; Teixeira de Sousa, Jr. P. Flavonoids from Combretum lanceolatum Pohl. Biochem. Syst. Ecol., 2013, 49, 37-38.

[53] Wu, M.M.; Wang, L.Q.; Hua, Y.; Chen, Y.G.; Wang, Y.Y.; Li X.Y.; Li, Y.; Li, T.; Yang, X.Y.; Tang, Z.R. New chalcone and dimeric chalcones with 1,4-p-benzoquinone residue from Combretum yunnanense. Planta Med., 2011, 77(5), 481-484

[54] Wang, L.Q.; Wu, M.M.; Liu, J.P.; Li, Y.; Hua, Y.; Wang, Y.Y.; Li, X.Y.; Chen, Y.G.; Wang, J.H. Five new diarylpropan-1-ols from Combretum yunnanense. Planta Med., 2011, 77(16), 1841-1844.

[55] Moosophon, P.; Kanokmedhakul, S.; Kanokmedhakul, K. Diarylpropanes and an arylpropyl quinone from Combretum griffithii. J Nat Prod., 2011, 74(10), 2216-2218.

[56] Kovács, A.; Vasas, A.; Hohmann, J. Natural phenanthrenes and their biological activity. Phytochemistry, 2008, 69 (5), 1084-1110.

[57] Letcher, R.M. and Nhamo, L.R.M.; Chemical constituents of the combretaceae. Part IV. Phenanthrene derivatives from the heartwood of Combretum hereroense. J. Chem. Soc., Perkin Trans. 1, 1973, 1179-1181.

[58] Pettit, G.R.; Singh, S.B.; Niven, M.L.; Hamel, E.; Schmidt, J.M. Isolation, structure, and synthesis of combretastatins A-1 and B-1, 
potent new inhibitors of microtubule assembly, derived from Combretum caffrum. J. Nat. Prod., 1987, 50(1), 119-131.

[59] Schwikkard, S.; Bing-Nan, Z.; Glass, T.E.; Sharp, J.L.; Mattern, M.R.; Randall, K.J.; Kingston, D.G.; Bioactive compounds from Combretum erythrophyllum. J. Nat. Prod., 2000, 63, 457-560.

[60] Pettit, G.R.; Singh, S.B.; Schmidt, J.M.; Niven, M.L.; Hamel, E.; Lin, C.M. Isolation, structure, synthesis, and antimitotic properties of combretastatins B-3 and B-4 from Combretum caffrum. J. Nat. Prod., 1988, 51(3), 517-527.

[61] Eloff, J.N.; Famakin, J.O.; Katerere, D.R.P. Isolation of antibacterial stilbene from Combretum Woodii (Combretaceae) leaves. Afr. J. Biotechnol., 2005, 4(10), 1167-1171.

[62] Letcher, R.M.; Nhamo, L.R.M.; Gumiro, I.T. Chemical constituents of the Combretaceae. Part II. Substituted phenanthrenes and 9,10-dihydrophenanthrenes and a substituted bibenzyl from the heartwood of Combretum molle. J. Chem. Soc., Perkin Trans. 1, 1972, 206-210.

[63] Letcher, R.M. and Nhamo, L.R.M. Chemical constituents of the combretaceae. Part III. Substituted phenanthrenes, 9,10dihydrophenanthrenes, and bibenzyls from the heartwood of Combretum psidioides. J. Chem. Soc., Perkin Trans. 1, 1972, 29412946.
[64] Malan, E.E.; Swinny, E. Substituted bibenzyls, phenanthrenes and 9,10-dihydrophenanthrenes from the heartwood of Combretum apiculatum. Phytochemistry, 1993, 34(4), 1139-1142.

[65] Adnyana, I.K.; Tezuka, Y.; Awale, S.; Banskota, A.H.; Tran, K.Q.; Kadota, S. 1-O-galloyl-6- $O$-(4-hydroxy-3,5-dimethoxy)benzoylbeta- $D$-glucose, a new hepatoprotective constituent from Combretum quadrangulare. Planta Med., 2001, 67(4), 370-371.

[66] Asami, Y.; Ogura, T.; Otake, N.; Nishimura, T.; Xinsheng, Y.; Sakurai, T.; Nagasawa, H.; Sakuda, S.; Tatsuta, K. Isolation and synthesis of a new bioactive ellagic acid derivative from Combretum yunnanensis. J. Nat. Prod., 2003, 66(5), 729-31.

[67] Brookes, K.B.; Doudoukina, O.V.; Katsoulis, L.C.; Veale, D.J.H Uteroactive constituents from Combretum kraussii. S. Afr. J. Chem., 1999, 52, 127-132.

[68] Jossang, A.; Pousset, J.L.; Bodo, B. Combreglutinin, a Hydrolyzable Tannin from Combretum glutinosum. J. Nat. Prod., 1994, 57(6), $732-737$.

[69] Katerere, D.R.; Gray, A.I.; Kennedy, A.R.; Nash, R.J.; Waigh, R.D. Cyclobutanes from Combretum albopunctatum. Phytochemistry, 2004, 65(4), 433-438.

(C) Dawe et al.; Licensee Bentham Open.

This is an open access article licensed under the terms of the Creative Commons Attribution Non-Commercial License (http://creativecommons.org/licenses/by-nc/3.0/) which permits unrestricted, non-commercial use, distribution and reproduction in any medium, provided the work is properly cited. 